\title{
Review of the species of the sylvicola group within Corticaria Marsham, 1802 (Coleoptera: Latridiidae) of Mediterranean region
}

\author{
José C. Otero \& Wolfgang H. Rücker
}

\begin{abstract}
Otero, J. C. \& Rücker, W. H. 2017: Review of the species of the sylvicola group within Corticaria Marsham, 1802 (Coleoptera: Latridiidae) of Mediterranean region. - Enomol. Fennica 28: 75-102.
\end{abstract}

A systematic review of the Mediterranean region species of the sylvicola group of Corticaria Marsham, 1802 is carried out. An identification key for the determination of those 21 species is provided. Morphological structures of the studied species are re-appraised and figures are provided of head, antennae, pronotum, and aedeagus with internal armature. The true identity of Corticaria tunisiensis Brisout de Barneville, 1884 is established by examination of the specimens of the collection of National Museum of Natural History, Paris, here designating a male lectotype and paralectotypes of both sexes.

J. C. Otero, Departamento de Zoología y Antropología Física, Universidad de Santiago de Compostela, 15782 Santiago de Compostela, Spain; E-mail: josecarlos.otero@usc.es

W. H. Rücker, Von-Ebner-Eschenbach-Strasse 12, D-56567 Neuwied, Germany; E-mail:post@latridiidae.de

Received 29 November 2016, accepted 24 January 2017

\section{Introduction}

Deciding which species of the Corticariinae should be assigned to the genus Corticaria Marsham, 1802 has always proved a relatively easy task due to the peculiar combination of characters identifying this relatively homogeneous taxon. However, species-level assignment of the large number of taxa within the genus is typically very difficult, generally requiring a study of diverse anatomic and secondary sexual traits of several specimens. In the view of this situation, Belon (1897) proposed the use of nine species groups within five subgenera, characterized by a few easily determined discriminating features. The proposal to use subgenera within this morphologically homogeneous genus has been dis- carded, but the species groups have remained a useful taxonomic device.

One of Belon's groups was created around the species Corticaria sylvicola Brisout, 1863. The members of this "sylvicola group" are characterized by their restricted Mediterranean distribution (Greece, Iberian Peninsula, North Africa, South France and Turkey), and by some very particuliar and constant traits, namely: 1) the elytral pubescence is long and straight, combined with the pronotal pubescence being long and erect; 2) the body is short, strongly convex, and reddish-brown; 3) the sides of the pronotum are strongly denticulate; 4) the elytral striae are regular, and less than twice as long as wide; 5) the metasternum is always shorter than the ventrite 1 ; and 6) the species are faintly winged or apterous. 
The total number of specimens of this group previously cited from Iberia, France, Greece, Turkey and North Africa is very small, and insufficient to allow accurate assessment of geographical distributions in these regions. However, some very valuable studies have been published by the authors like Dajoz (1969, 1970), Otero \& Díaz Pazos (1986), Otero et al. (2006, 2013), Mariño et al. (2007), Otero \& López (2009, 2016), Rücker (2013a, b), and Reike (2015), providing the basis for current knowledge of these beetles in the Mediterranean region, which is reviewed here.

\section{Methods}

The terminology and measurements for the discussed species follow Otero $(1997,2001)$. The structures were measured under a Leica M205C stereomicroscope equipped with an analysis system Application Suite.

The following abbreviations are used below:

E, Eye's eccentricity = width $/ 1 / 2$ length; L, Length in dorsal view; WL, ratio width/ length; $\varnothing$, diameter.

BMNH, British Museum of Natural History, London, UK; HNHM, Hungarian Natural History Museum (Magyar Természettudományi Múzeum), Budapest, Hungary; MMCJ, Manchester Museum, Manchester, coll. C. Johnson; MNCN, Museo Nacional de Ciencias Naturales, Madrid, Spain; MNHG, Muséum d'Histoire Naturelle, Geneva, Switzerland; MNHN, Muséum National d'Histoire Naturelle, Paris, France; MNHUB, Museum für Naturkunde, Humboldt-Universität zu Berlin, Germany; MZB, Museu de Zoología, Barcelona, Spain; MZLU, Museum of Zoology and Entomology, Lund University, Sweden; NMW, Naturhistorisches Museum Wien, Vienna, Austria; UMC, coll. Universidad de Murcia, Spain; USC, Universidad de Santiago de Compostela, Spain, coll. J. C. Otero; WRC, coll. Wolfgang H. Rücker, Neuwied, Germany

\section{Species of Corticaria of the sylvicola group of Mediterranean region}

\subsection{Corticaria abdominalis Dajoz (Fig. 1)}

\section{Corticaria abdominalis Dajoz 1970}

Type material examined. Holotype (ठ): Spain, León, Sierra de Son, San Feliz de las Lavanderas (leg. H. Franz) (NMW). Paratypes (7 $q$ ) Spain. León, Sierra de Son, San Feliz de las Lavanderas, 5 우 (leg. H. Franz). León, Sierra de Cabrera, Molinaferreda, 2 q $q$ (leg. H. Franz) (NMW).

Other specimens examined. Spain. Lugo, Sierra de los Ancares, Cabana Vella, 8.X.1984, $7 \widehat{\jmath} \widehat{\jmath}$ and 2 우. Lugo, Sierra de los Ancares, Cela, IX.1984, 1 $\lesssim$ and 1 q. Lugo, Sierra de los Ancares, Os Cabaniños, 8.X.1984, 12 $\widehat{\jmath}$ and 3 우. Lugo, Sierra del Caurel, Rugueira, 8.IX. 1985, 2 §ิ ô. Lugo, Sierra del Caurel, Seoane, 8.IX.1985, 19 exx, Zamora, Sierra de Segundera, Porto, IX.1983, 2 exx (leg. J. C. Otero) (USC).

Morphology. Body. Length 1.8-2.0 mm (Fig. 1a). Well-sclerotized specimens bright brown, less sclerotized ones amber yellow; legs slightly lighter than rest of body. Elytra thickly punctured. White pubescence, short $(\mathrm{L}=50-69 \mu \mathrm{m})$, little erected, almost absent in interstriae. Metathoracic wings absent.

Head. Transverse (WL $=1.7-1.8)$ with strongly reticulate tegument. Cephalic puncturation deep and spaced. Eyes medium-sized ( $\mathrm{L}=$ 93-110 $\mu \mathrm{m}$ ), made up of few thick facets, slightly convex and moderately protuberant $(\mathrm{E}=0.9)$. Temples as long as half eye. Antennae (Fig. 1b) long $(\mathrm{L}=0.630-0.650 \mathrm{~mm})$. All antennomeres longer than broad except segment $\mathrm{X}$ as long as broad. Antennomeres VI and VII strongly narrowed at their base.

Pronotum (Fig. 1a). Moderately transverse $(\mathrm{WL}=1.5-1.6)$, convex with a basal pit slightly marked. Lateral margins rounded and slightly denticulate, except denticle of posterior angle generally larger than rest. Puncturation compact and dense $(\varnothing=14-18 \mu \mathrm{m})$, dorsal surface reticulate. Scutellar shield transverse and flat. Elytra together very convex and only 1.4 times longer than broad. Lateral edge slightly marked and only visible on anterior quarter. Striae made up of strong 
Fig. 1. Corticaria abdominalis Dajoz. - a. Dorsal habitus. - b. Antenna. - c. Protibiae. -d. Ventrite 5, male. $-\mathrm{e}$. Aedeagus, dorsal

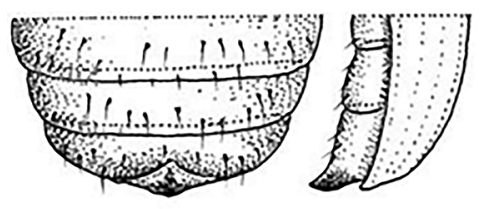

d

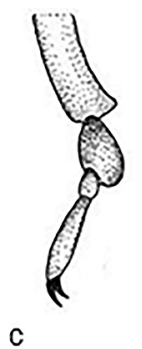
view. - f. Aedeagus, lateral view.
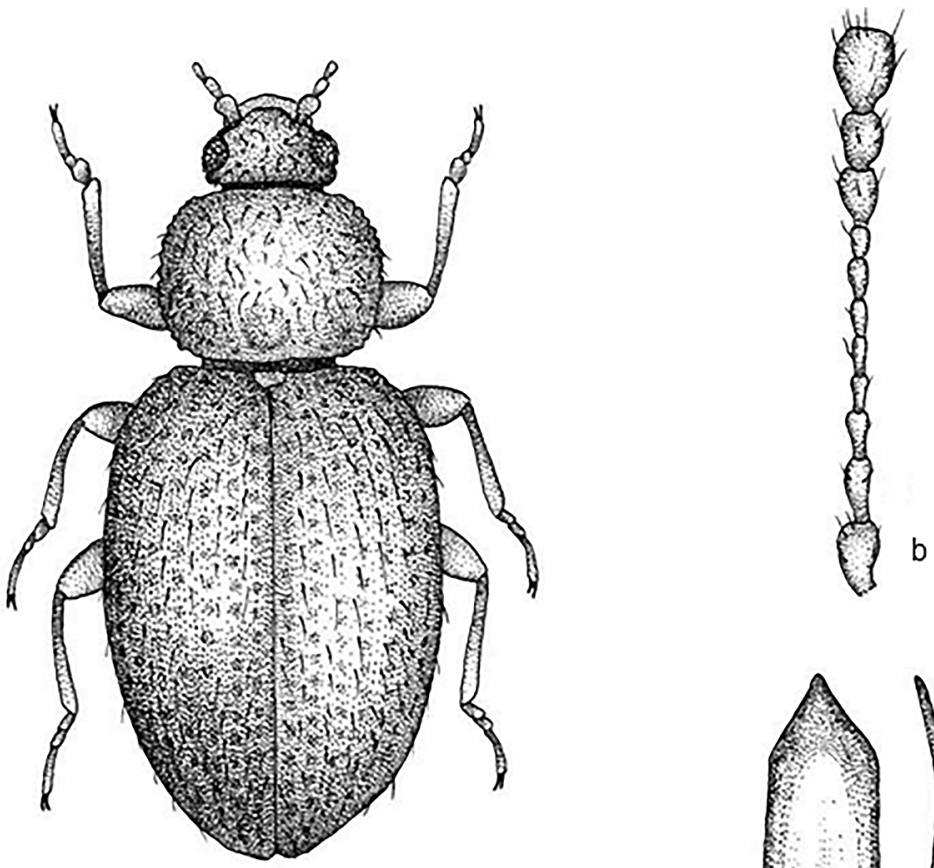

a

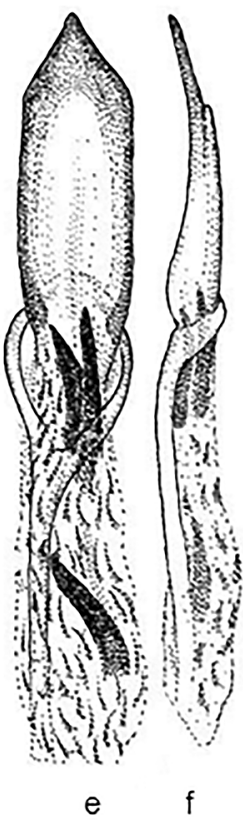

puncturation $(\varnothing=18-22 \mu \mathrm{m})$. Prosternum with two pits located in front of anterior coxae. Metasternum short, as long as two thirds of that of first abdominal segment. Anterior and medial tibiae with a denticle in inner apical angle in males (Fig. 1c). Female tibiae lacking spines.

Abdomen. Slightly pubescent. Ventrite 5 of male (Fig. 1d) prolonged in a rounded and forward-curved tip, whereas that of female flat.

Aedeagus (Fig. 1e, f). Elongated $(\mathrm{L}=0.750$ $\mathrm{mm})$, broad with a sharp tip. Internal sac with four large $\operatorname{rods}(\mathrm{L}=0.110-0.180 \mathrm{~mm})$ and a large number of small needle-shaped ones.
Distribution. NW Spain (Johnson 2007, Rücker 2013a).

Biology. Unknown.

\subsection{Corticaria anatolica C. Johnson (Fig. 2)}

Corticaria anatolica C. Johnson 1989: 79

Type material examined. Paratype (ð): Turkey, Antalya, Antalya-Kemer, 4.V.1975 (leg. C. Besuchet and I. Löbl) (MMCJ).

Morphology. Body. Length 1.4-1.7 mm, oval and convex (Fig. 2a). Entirely pale reddish 


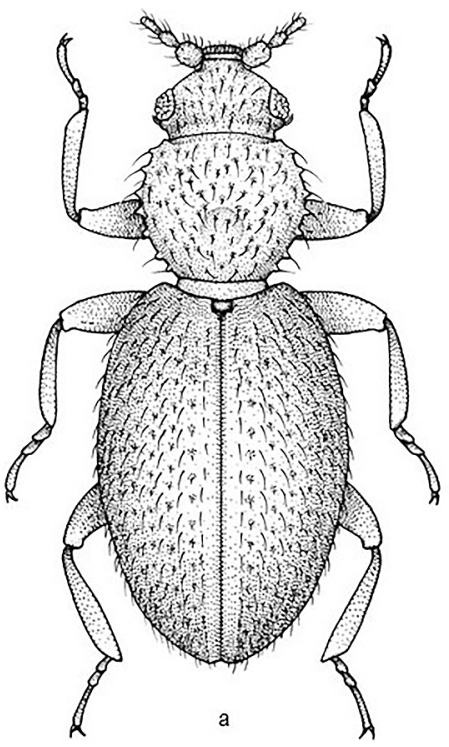

brown, legs and antennae lighter. Pubescence double $(\mathrm{L}=34-74 \mu \mathrm{m})$; interstitial hairs semierect, hairs mostly nearly straight and bristling, separated by almost their length, not over-lapping; strial hairs over twice as numerous but only half length of interstitial hairs, somewhat raised, slightly curved, not overlapping; striae somewhat strongly and closely punctured; interstices very narrow at base; widening towards basal third where slightly wider than striae, their punctures fine and sparse. Metathoracic wings absent.

Head. Only a little narrower than pronotum, little transverse (WL $=1.4-1.5$ ). Labrum large and rounded in anterior angles. Temples well marked, somewhat short, about a third length of eyes, distinctly widened behind, clearly obtusely angulate; eyes distinctly wider than temples, but only moderately so, little protuberant $(\mathrm{E}=0.7$ 0.8 ). Reticulation distinct, puncturation moderate in size and closeness. Antennae (Fig. 2b) moderately long $(\mathrm{L}=0.49-0.55 \mathrm{~mm})$, club abrupt, rather short; stem antennomeres short; antennomere VIII broader than long, IX distintctly, $\mathrm{X}$ more obviously, broader than long.

Pronotum (Fig. 2a). Rather small and somewhat narrow, broadest about middle, slightly heart-shaped and rather square (WL $=1.2-1.3$ ); sides moderately curved, with six to seven coarse crenulations, sometimes with more feeble and effaced crenulations, sometimes with more feeble and effaced crenulations between them; front and
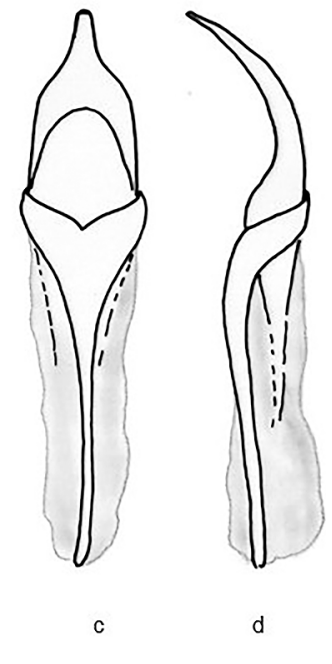

Fig. 2. Corticaria ana-

tolica Johnson. - a.

Dorsal habitus. - b. Antenna. - c. Aedeagus, dorsal view. $-\mathrm{d}$.

Aedeagus, lateral view.

hind angles strongly toothed due to size and shape of crenulations, each angle with a long and almost straight, outstanding seta of length 0.080-0.095 $\mathrm{mm}$; lateral pubescence short and fine, swept along sides and barely projecting; surface rather shining, microsculpture present but usually obscured; punctures moderately large and impressed $(\varnothing=18-20 \mu \mathrm{m})$; disc convex; post median depression strong, lateral impressions absent. Elytra together short oval, 2.50-2.57 times as long as pronotum, 1.49-1.54 times as long as broad; more convex and more ample than pronotum, breadth $0.6-0.7 \mathrm{~mm}$; sides moderately curved, almost straight-sided in middle third, similarly curved both apically and basally, distinctly serrate along basal third at least. Prosternum with pubescent transverse fovea anterolateral to procoxae. Mesocoxae very narrowly separated by acuminate process. Metasternum short. Legs moderately long. Protibiae and mesotibiae with a forked tooth on inner edge at apex; protarsi with basal segment only slightly dilated.

Abdomen. Ventrite 1 somewhat finely and sparsely punctured; ventrite 5 simple.

Aedeagus (Fig. 2c, d). Strangulated in anterior third with elongate apical end.

Distribution. SE Turkey (Johnson 2007, Rücker 2013a).

Biology. Unknown. 
Fig. 3. Corticaria antonioi Otero, López \& Rücker. - a. Dorsal habitus. - b. Antenna. - c. Punctures. - d. Aedeagus, dorsal view. - e. Aedeagus, lateral view.

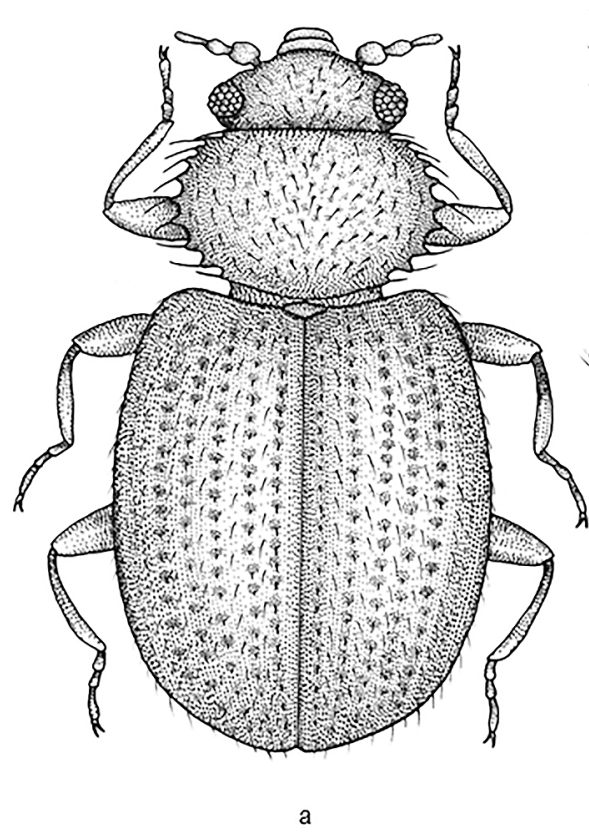

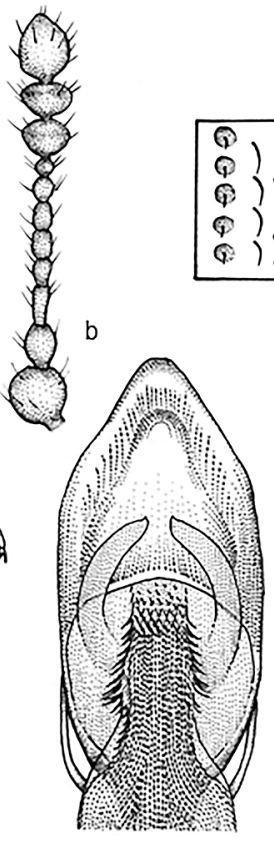

d

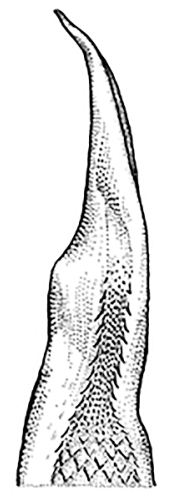

e

\subsection{Corticaria antonioi Otero, López \& Rücker} (Fig. 3)

\section{Corticaria antonioi Otero, López \& Rücker} 2013: 237

Type material examined. Holotype (ठ): Spain, Murcia, Moratalla, El Sabinar [38 $12^{\prime} 17^{\prime}$ N 2109'17’ W; 8.VI.1979] (leg. J.J. Presa) (USC).

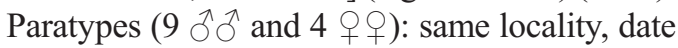
and collector as the Holotype. $1 \lambda$ and 1 (WRC); $1 \hat{\delta}$ and $1 \%(\mathrm{MNCN}) ; 1 \hat{\delta}$ and $1 \%$ (MZB); $1 \delta^{\lambda}$ and 19 (UMC); others in coll. Otero (USC).

Morphology. Body. Length 1.6-1.7 mm, short, oval and convex (Fig. 3a). Some specimens are yellowish-brown, others ferruginous-brown. Pubescence yellowish, long $(\mathrm{L}=55-71 \mu \mathrm{m})$ and erect. Metathoracic wings absent.

Head. Inclined, little transverse (WL $=1.3-$ 1.4) and with rough surface. Labrum large, rounded in anterior angles. Eyes hemispheric, large $(\mathrm{L}=0.114 \mathrm{~mm})$, scarcely protuberant $(\mathrm{E}=$ $0.9)$. Ocular facets $(\varnothing=16-18 \mu \mathrm{m})$ smaller than punctures of head $(\varnothing=21-27 \mu \mathrm{m})$. Antennae (Fig. 3b) narrow, pubescent and short $(\mathrm{L}=0.483$ $\mathrm{mm}$ ) not reaching base of pronotum. Antenno- meres III to VII longer than broad; VIII as long as broad. Antennal club elongated; IX and $\mathrm{X}$ antennomeres transverse and equal in length.

Pronotum (Fig. 3a). Very convex, cordiform and moderately transverse ( $\mathrm{WL}=1.5-1.6)$; narrower in base than in apex; widest slightly at front of middle. Anterior margin straight. Anterior angles rounded, posterior angles obtuse. Lateral margins strongly denticulated in all their length. Prebasal pit absent. Punctures not very deep, separated by a distance longer than their diameter $(\varnothing$ $=20-21 \mu \mathrm{m})$ (Fig. 3c). Scutellar shield transverse. Elytra together 3.5 times longer than pronotum. Pilosity double and long. Anterior tibiae straight in their internal margin and with a terminal spine, cut obliquely in apex of their external margin. $1^{\text {st }}$ tarsomere dilated and pubescent underneath.

Abdomen. Ventrite 5 as long as two following ones together, ventrite 5 flat and with some lines of setae in its apical margin.

Aedeagus (Fig. 3d, e). Triangular and with apex rounded. Endophallic apodeme formed by two long sclerotized rods that look like a "( )". Base of aedeagus with numerous small needleshaped sclerotized rods. Aedeagus forms an obtuse angle in its apical portion in lateral view. 


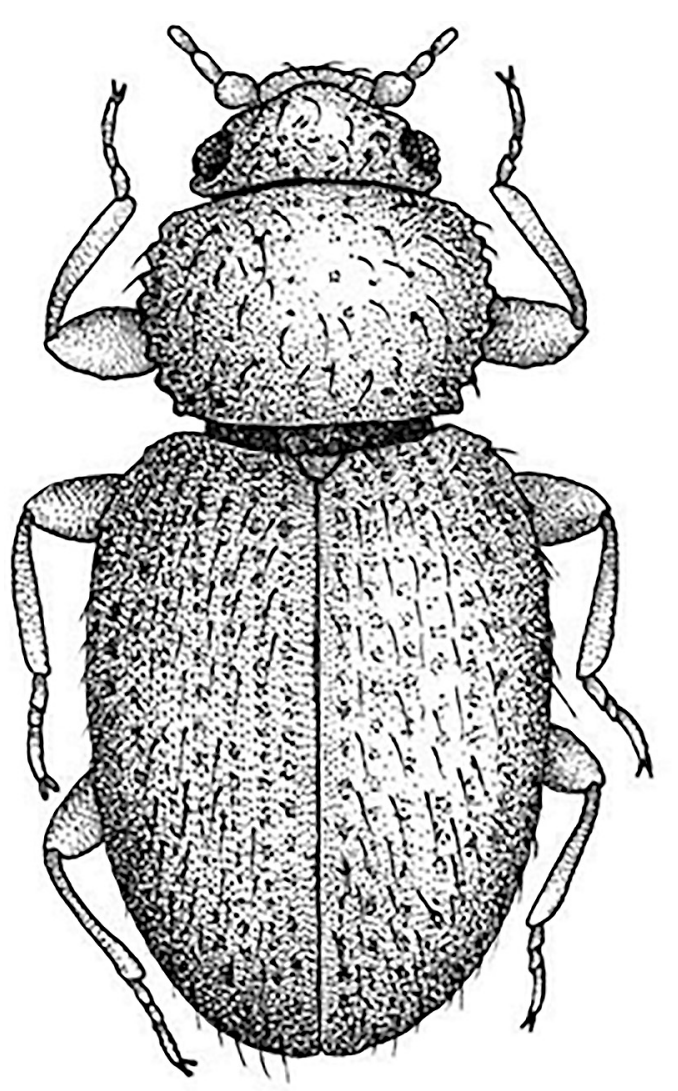

a

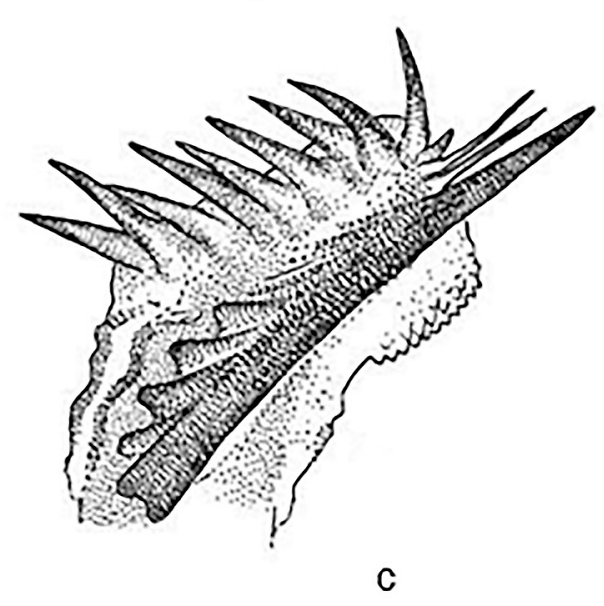

Female. Same external morphological characters as male.

Distribution. SE Spain (Otero et al. 2013).

Biology. Captured from among fallen needles of Juniperus sp.
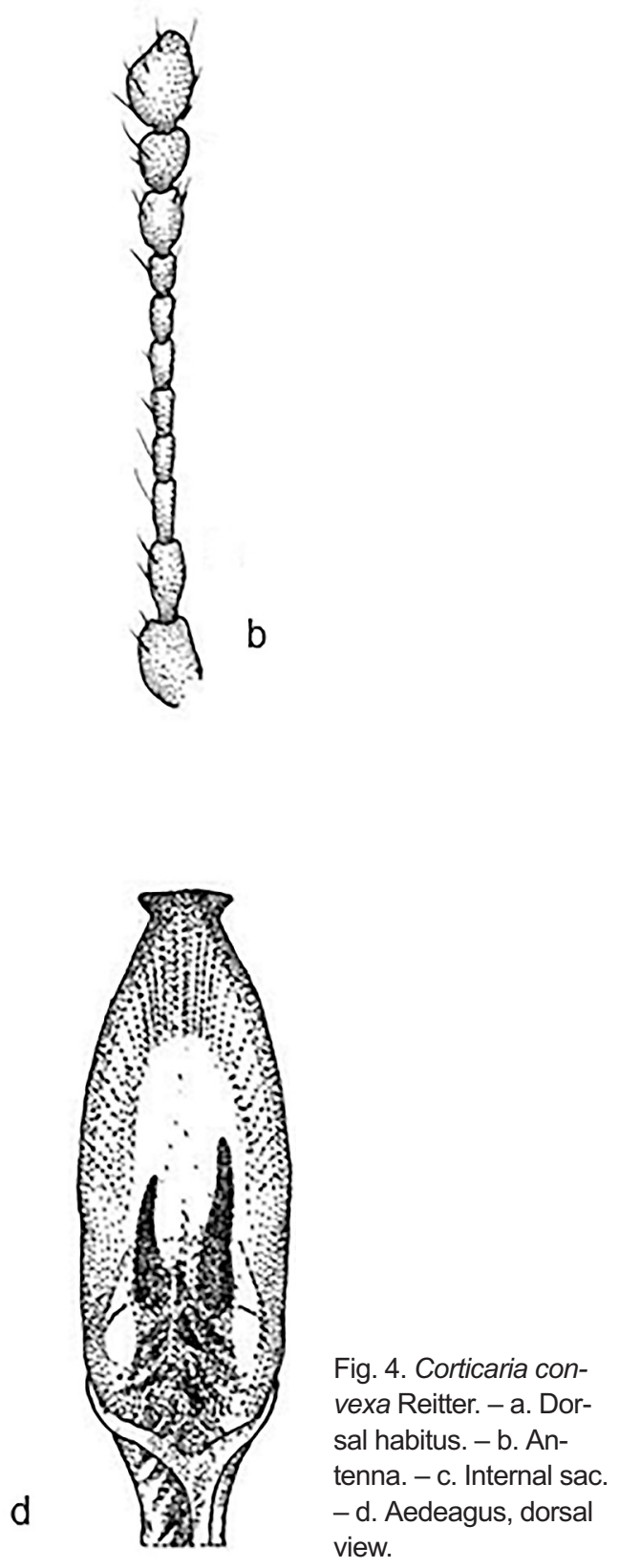

3.4. Corticaria convexa Reitter (Fig. 4)

Corticaria convexa Reitter 1881: 60

Type material examined. Holotype (ふ): Algeria, Desbrochens des Logues, 1881 (leg. E. Reitter) (HNHM). Paratypes (1 $\hat{\sigma}$ and $3 \hat{q}+$ ): Algeria, 
Fig. 5. Corticaria cotovillae Otero \& Díaz

Pazos. - a. Dorsal habitus. - b. Antenna. $-c$. Aedeagus, dorsal view. - d. Aedeagus, lateral view.

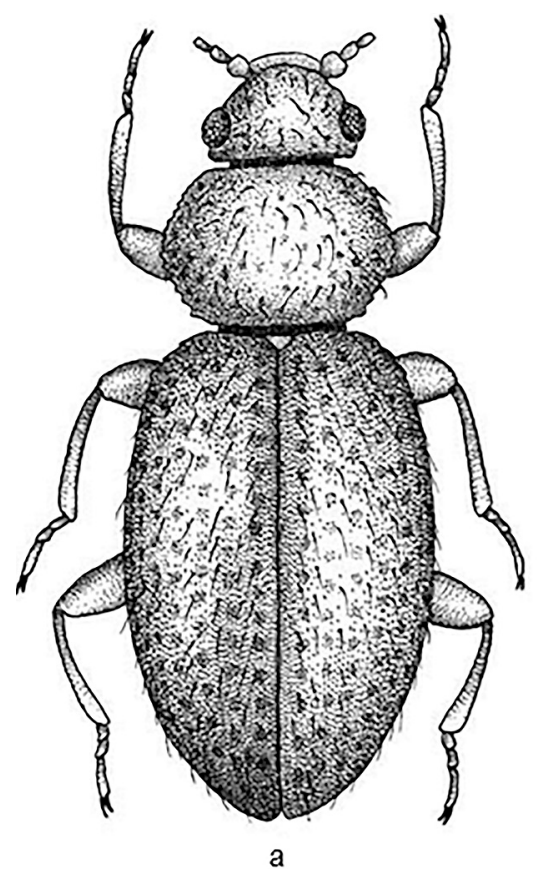

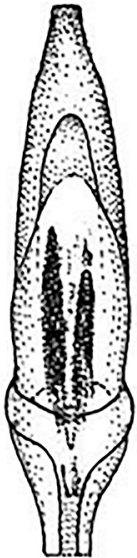

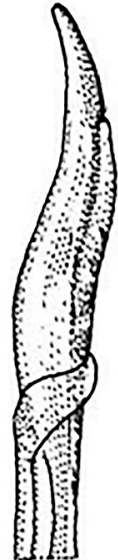

c

d
Bou Berak, Kabilie L. Puel (leg. E. Reitter) (HNHM).

Others specimens examined: Spain, Madrid, El Escorial, 1 ภ (MNCN). Salamanca, El Cerro, 24.V.1980, 1 đิ (leg. R. Outerelo) (USC).

Morphology. Body. Length 1.8-2.0 mm, very convex and oval (Fig. 4a). Colouration reddishbrown. Pubescence long $(\mathrm{L}=75-110 \mu \mathrm{m})$ and white. Metathoracic wings absent.

Head. Very transverse $(W L=1.7-1.8)$, semicircular. Cephalic punctures wide $(\varnothing=18 \mu \mathrm{m})$ and deep. Eyes conspicuous $(\mathrm{L}=0.110 \mathrm{~mm})$, slightly prominent $(E=0.9)$. Temples wellmarked, one third of eye's length. Antennae (Fig. 4c) long $(\mathrm{L}=0.630-0.640 \mathrm{~mm})$ and pubescent. Antennomeres I to VI clearly longer than broad; VII and VIII progressively sub-squared; IX and XI, 0.5 times longer than broad; X segment transverse.

Pronotum (Fig. 4a). Very robust, slightly heart-shaped and rather square $(\mathrm{WL}=1.4)$, broadest in its anterior third, with front and back edges of similar width. Lateral margins with 7-8 well-marked denticles. Post-median depression marked. Pronotal puncturation strong $(\varnothing=30-35$ $\mu \mathrm{m})$ and dispersed. Scutellar shield transverse, flat. Elytra together oval and very short, only 1.3 times longer than broad and 2 times longer than pronotum. Lateral margin well-marked in anterior third. Elytral puncturation strong $(\varnothing=30-35$ $\mu \mathrm{m})$ and irregular. Femora greatly widened. Tibiae with spine on internal apical angle in males; also present in females but less apparent.

Aedeagus (Fig. 4d). Lance-shaped with truncated apex, showing a regular curvature in lateral view. Internal sac (Fig. 4c) with a long spine ( $\mathrm{L}=$ $0.500 \mathrm{~mm}$ ) and two rows of smaller rods: first row comprising conspicuous spines arranged in a curve originating in central spine, second row comprising small rods located between spine and first row.

Distribution. Spain, N Algeria (Johnson 2007, Rücker 2013a).

Biology. The examined specimens have been collected from remains of Quercus sp. and Castanea sp.

\subsection{Corticaria cotovillae Otero \& Díaz Pazos} (Fig. 5)

Corticaria cotovillae Otero \& Díaz Pazos 1986: 167

Type material examined. Holotype (ð): Spain, Madrid, Montejo de la Sierra, 2.VII.1972 (leg. F. Novoa) (USC). Paratypes ( 2 + $)$ ): Spain, Mad- 
rid, Montejo de la Sierra, 1.V.1972; 24.VII.1972 (leg. F. Novoa).

Morphology. Body. Length 1.8-2.0 mm, convex and oval (Fig. 5a). Entirely testaceous yellow. Pubescence $(\mathrm{L}=65-72 \mu \mathrm{m})$ overlapping. Metathoracic wings absent.

Head. Very transverse $(\mathrm{WL}=2.0)$. Temples little angular, slightly longer than eye. Surface slightly punctured and strongly reticulate in posterior half of head. Eyes scarcely protuberant $(\mathrm{E}=$ $0.5)$, with few thick and scarce facets ( 6 or 7 along whole eye length). Antennae (Fig. 5b) $(\mathrm{L}=0.665$ $\mathrm{mm}$ ) short, reaching posterior margin of pronotum. Antennomere I thick and spherical; antennomere II narrower and elongated; from III to VII longer than broad; VIII almost as wide as long. Three last antenomeres forming an elongated club, longer than broad.

Pronotum (Fig. 5a). Moderately transverse (WL $=1.4-1.5)$, cordiform, convex without basal pit. Greatest width approximately at midpoint. Lateral denticulation strong, above all in posterior half. Puncturation strong $(\varnothing=20-26 \mu \mathrm{m})$ but spaced. Scutellar shield transverse and flat. Elytra together ovoid, 1.4 to 1.5 times wider than pronotum and almost 1.5 times longer than broad. Lateral margin visible only in anterior third. Striae strongly punctured with thick punctures $(\varnothing=38$ $46 \mu \mathrm{m}$ ) separated by a longer distance than their diameter. Prosternum with two pits in front of an- terior coxae. Anterior tibiae straight and cut obliquely inapex of their external margin. First segment of tarsi dilated and ciliated underneath.

Abdomen. Covered with a fine pubescence. Ventrite 11.4 times longer than metasternum.

Aedeagus (Fig. 5c). Length $0.690 \mathrm{~mm}$, truncated in apex; slightly and regularly curved in its apical half in lateral view (Fig. 5d). Internal sac with numerous small needle-shaped rods.

Distribution. Central Spain (Otero \& Díaz Pazos 1986, Johnson 2007, Rücker 2013a).

Biology. Unknown.

\subsection{Corticaria cretica C. Johnson (Fig. 6)}

Corticaria cretica C. Johnson 1989: 80

Type material examined. Paratype ( $(+)$ : Greece, Crete, Kalo Chorio, 3.VIII.1982 (Leg. S. Vit) (MMCJ).

Morphology. Body. Length 1.4-1.5 mm, moderately convex (Fig. 6a). Entirely pale reddish brown, legs and antennae lighter. Pubescence double; interstitial hairs semi-erect, mostly almost straight and bristling (male) or slightly more curved (female), length $0.08 \mathrm{~mm}$, separated by their length, not or barely overlapping, nearly twice as numerous as strial hairs, almost half length of interstitial hairs, somewhat raised, slightly curved, not or barely overlapping; striae

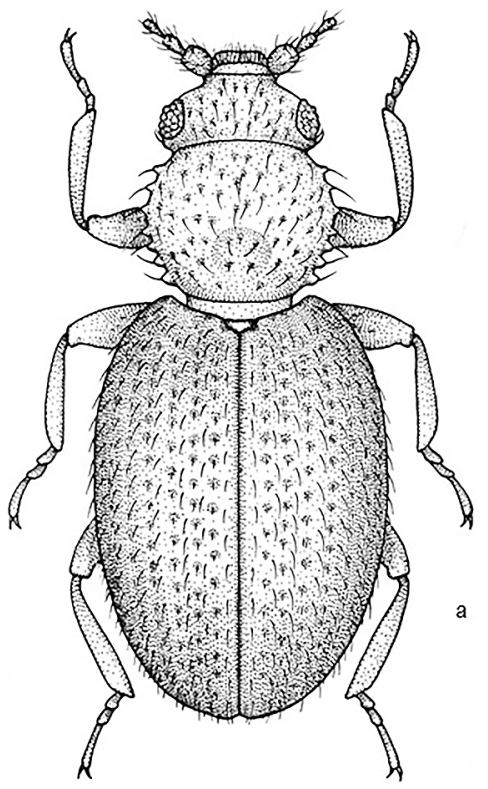

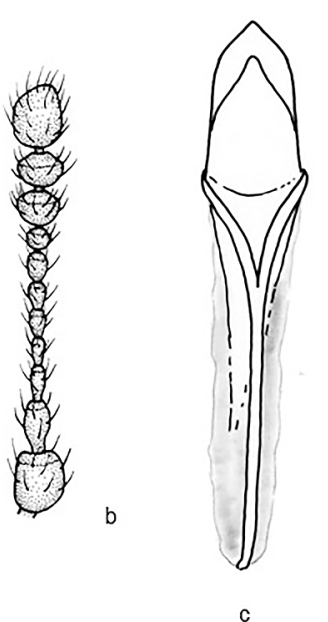

Fig. 6. Corticaria cretica Johnson. - a. Dorsal habitus. - b. Antenna. - c. Aedeagus, dorsal view. - d. Aedeagus, lateral view. 


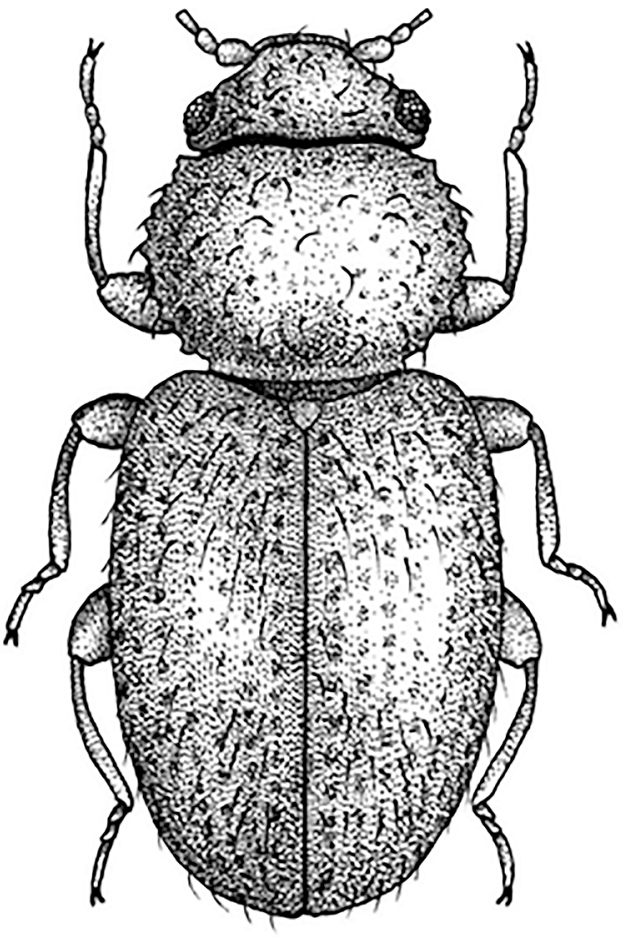

Fig. 7. Corticaria diecki Reitter. - a. Dorsal habitus. - b. Antenna. - c. Internal sac. - d. Aedeagus, dorsal view. - e. Aedeagus, lateral view. a

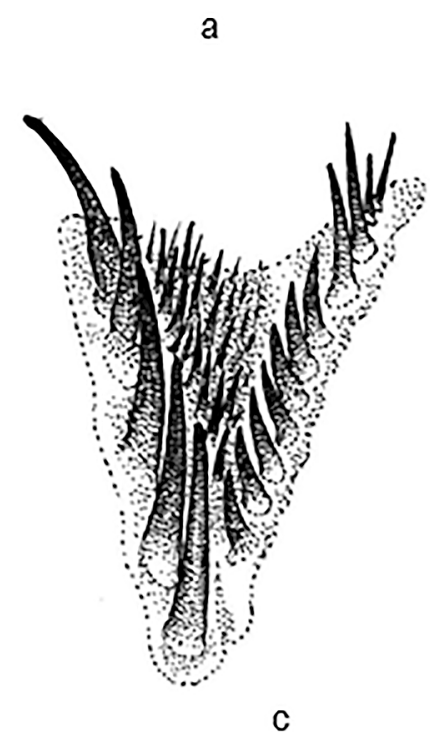

$d$
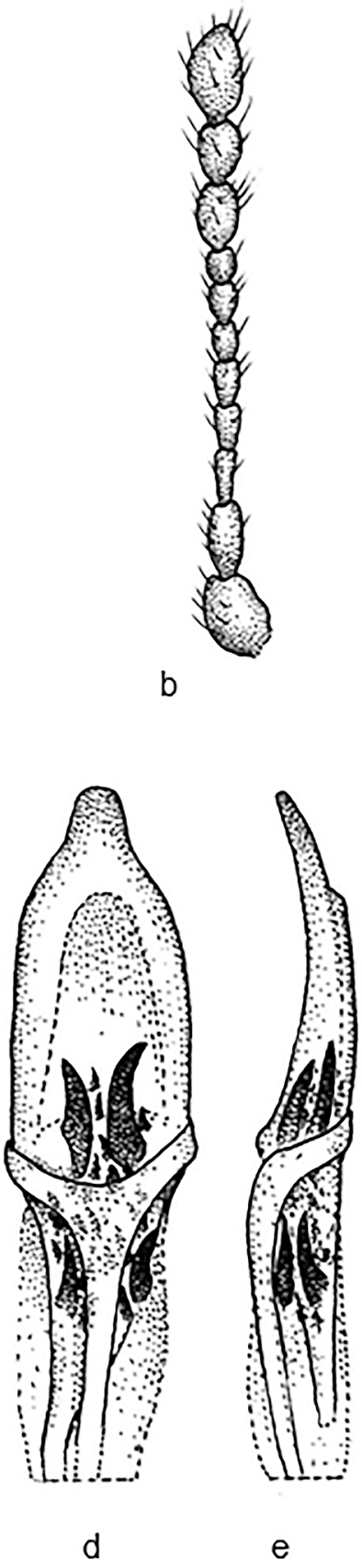

tion distinct, puncturation moderate in size and closeness $(\varnothing=18-20 \mu \mathrm{m})$; surface little shining. Eyes medium-sized $(\mathrm{L}=94 \mu \mathrm{m})$, slightly prominent $(E=0.9)$. Temples well marked, somewhat short, about a third of length of eyes, distinctly obtusely angulate. Antennae (Fig. 6b) moderately long $(\mathrm{L}=0.48-0.53 \mathrm{~mm})$, club abrupt and
Head. Only a little narrower than pronotum; moderately transverse $(\mathrm{WL}=1.5-1.6)$; reticula- moderately to somewhat strongly closely punctured; interstices somewhat narrower than striae at extreme base, thence rapidly widening their rather fine and sparse punctures. Metathoracic wings absent. moderately transverse (WL $=1.5-1.6) ;$ reticula- 
rather short; stem antennomeres short; antennomere VIII broader than long, IX clearly, X more obviously, broader than long.

Pronotum (Fig. 6a). Rather small, somewhat narrow, broadest about middle; moderately transverse $(\mathrm{WL}=1.5-1.6)$; sides moderately curved, with seven rather coarse crenulations and a few minor ones in between, front and hind angles clearly toothed due to size and shape crenulations, each angle with a long and almost straight, outstanding seta of length $0.080-0.095$ $\mathrm{mm}$; lateral pubescence much shorter and finer, swept along sides and barely projecting; surface rather shining, microsculpture present but not very distinct; punctures moderately large and impressed, rather close, abouth half a diameter apart on disc; disc convex; post median depression strong, lateral impressions absent. Elytra together short oval, 2.5-2.6 times as long as pronotum, $1.50-1.54$ times as long as broad; only slightly more convex but ampler than pronotum, breadth 0.62-0.64 mm; sides moderately curved, almost straight-sided in middle third, similarly curved both apically and basally, distinctly serrate within basal third. Prosternum with pubescent transverse fovea antero-lateral to procoxae. Mesocoxae very narrowly separated by acuminate process. Metasternum short. Male: pro and mesotibiae with a very small tooth on inner edge at apex; protarsi with basal segment only slightly dilated. Legs moderately long.

Abdomen. Ventrite 1 somewhat finely and sparsely punctured; ventrite 5 simple.

Aedeagus (Fig. 6c, d). Apex triangular, margins rounded.

Distribution. Greece (Crete Island: Johnson 2007, Rücker 2013a).

Biology. Unknown.

\subsection{Corticaria diecki Reitter (Fig. 7)}

Corticaria diecki Reitter 1875: 413

Corticaria kaufmanni Reitter 1881: 61

Type material examined. Types (3 exx): Morocco, Casablanca (leg. E. Reitter) (HNHM).

Other specimens examined. Spain, Andalucía, $1 \mathrm{\jmath}$. Morocco, Tangier, 1875, $1 \mathrm{~m}$ (leg. E. Reitter) (HNHM). As Corticaria kaufmanni Reitter, 1880. Algeria, Constantine,

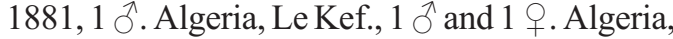
St. Charles, 2 우 (leg. E. Reitter) (HNHM).

Morphology. Body. Length 1.6-1.8 mm, short and very convex (Fig. 7a). Colouration reddish-brown. Pubescence yellowish, long and raised. Metathoracic wings absent.

Head. Semicircular, very wide and transverse $(\mathrm{WL}=1.9-2.0)$. Eyes medium-sized $(\mathrm{L}=80-85$ $\mu \mathrm{m})$, slightly prominent $(\mathrm{E}=0.45-0.5)$. Temples small, less than $1 / 5$ of eye's length, and scarcely prominent. Antennae (Fig. 7b) $(\mathrm{L}=0.500 \mathrm{~mm})$ almost reach posterior edge of pronotum. All antennomeres with low WL ratio, V, VI, VII and VIII being rounded; IX and $\mathrm{X}$ almost as long as broad, while XI is longer. Cephalic pubescence long $(\mathrm{L}=40-60 \mu \mathrm{m})$, straight and dense.

Pronotum (Fig. 7a). Rather heart-shaped, a little broader than long $(\mathrm{WL}=0.75)$ and as wide as elytra together. Lateral margins rounded, with 8-10 well-marked denticles. Post-median depression shallow. Pronotal puncturation rather fine $(\varnothing=20-25 \mu \mathrm{m})$. Pubescence long $(\mathrm{L}=60$ $100 \mu \mathrm{m})$, relatively raised and dense. Scutellar shield squared and flat. Elytra together sub-oval and very short $(\mathrm{WL}=0.7-0.8)$. Punctures of striae fine $(\varnothing=22-27 \mu \mathrm{m})$ and more compact than punctures of inter-striae. Pubescence very long $(\mathrm{L}=85-120 \mu \mathrm{m})$ and straight, but not dense.

Aedeagus (Fig. 7d, e). Lance-shaped, with a rounded apex. Internal sac (Fig. 7c) with two rows of rods: first row with 5-6 large curved rods, second one with 10 smaller spines; numerous small acicular pieces between the two rows.

Distribution. S Spain, N Algeria, N Morocco, N Tunisia (Johnson 2007, Rücker 2013a).

Biology. Unknown.

\subsection{Corticaria espanyoli Otero \& López} (Fig. 8)

Corticaria espanyoli Otero \& López 2009: 269

Type material examined. Holotype (ঐ): Spain, Barcelona, Berguedà, Espinalbet. 2.VI.1935. (leg. F. Español) (MZB).

Morphology. Body. Length 1.9-2.0 mm, convex and oval (Fig. 8a). Colouration reddishbrown. Pubescence long, white and raised. Metathoracic wings absent.

Head. Transverse $(\mathrm{WL}=1.7-1.8)$ with eyes medium-sized $(\mathrm{L}=90-95 \mu \mathrm{m})$ and moderately 


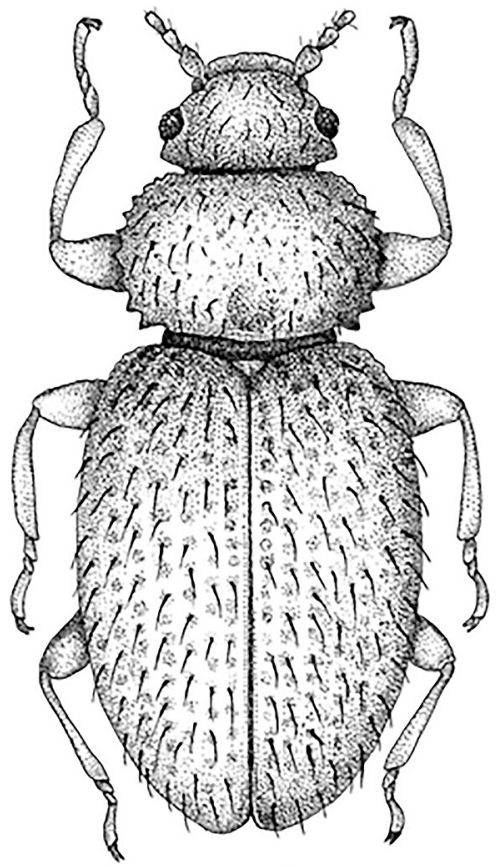

a

Fig 8. Corticaria espanyoli Otero \& López. - a. Dorsal habitus. - b. Antenna. - c. Ventrite 5 male. - d. Aedeagus, dorsal view. - e. Detail of the rod.

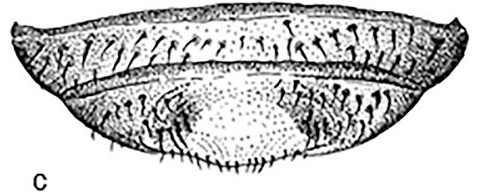

b
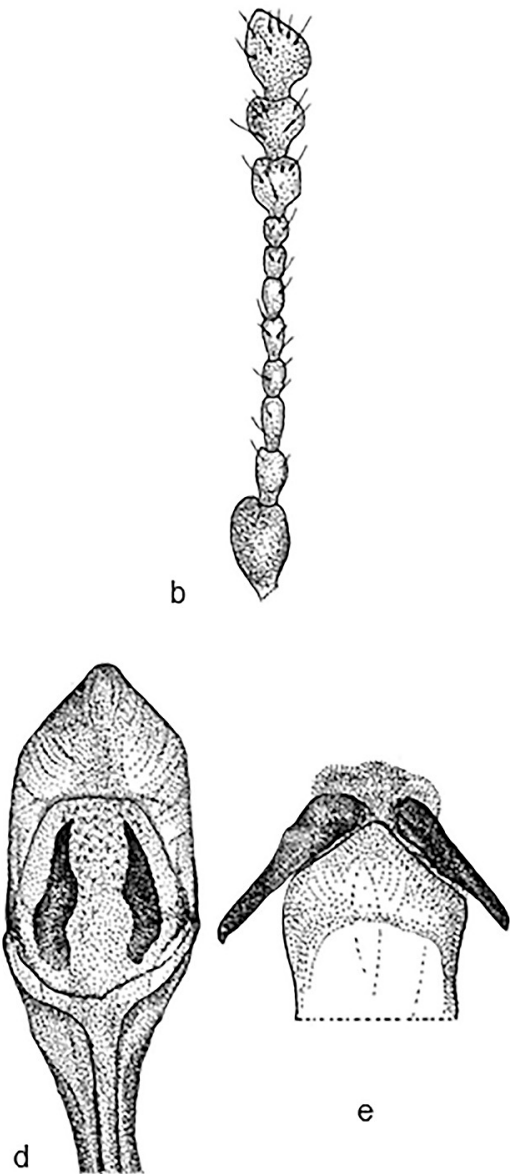

convex $(E=0.65)$. Temples large, almost $2 / 3$ of eye's length, angular, with scant pubescence. Medium-sized antennae $(\mathrm{L}=0.590 \mathrm{~mm})$ almost reaching posterior edge of pronotum. Antennomeres (Fig. 8b) I-VI clearly longer than broad; VII and VIII segments more rounded; IX sub-cyathiform; X clearly cyathiform and transverse. Antennomere XI trapezoidal. Cephalic puncturation shallow. Pubescence very long $(\mathrm{L}=50-90$ $\mu \mathrm{m})$, white and straight.

Pronotum (Fig. 8a). Disk-shaped, relatively transverse (WL $=1.5-1.6)$, as broad as elytra at their base. Lateral margins rounded and strongly denticulate, especially in posterior half. Post-median depression small and shallow. Pronotal puncturation strong and irregular $(\varnothing=25-35$ $\mu \mathrm{m})$. Pubescence somewhat shorter than cephalic pubescence $(\mathrm{L}=50-60 \mu \mathrm{m})$, white and very straight. Scutellar shield small, triangular and transverse. Elytra together oval, very short $(\mathrm{WL}=$ $0.8)$. Lateral edge slightly marked in anterior third. Punctures of striae broad $(\varnothing=34-36 \mu \mathrm{m})$ but superficial and badly aligned. Elytral pubescence very long $(\mathrm{L}=90-110 \mu \mathrm{m})$, straight and rather dense. Metasternum 1/3 shorter than ventrite 1 .

Abdomen. Ventrite 5 (Fig. 8c) with an apical concavity and a very marked pit, anteriorly almost reaching ventrite 4 .

Aedeagus (Fig. 8d). Lance-shaped, wide and very long $(\mathrm{L}=0.920 \mathrm{~mm})$. Anterior apex with a large fang-like $\operatorname{rod}(\mathrm{L}=0.180 \mathrm{~mm})$, totally protruded when internal sac is evaginated (Fig. 8e).

Distribution. NE Spain (Otero \& López 2009, Rücker 2013a).

Biology. Unknown. 


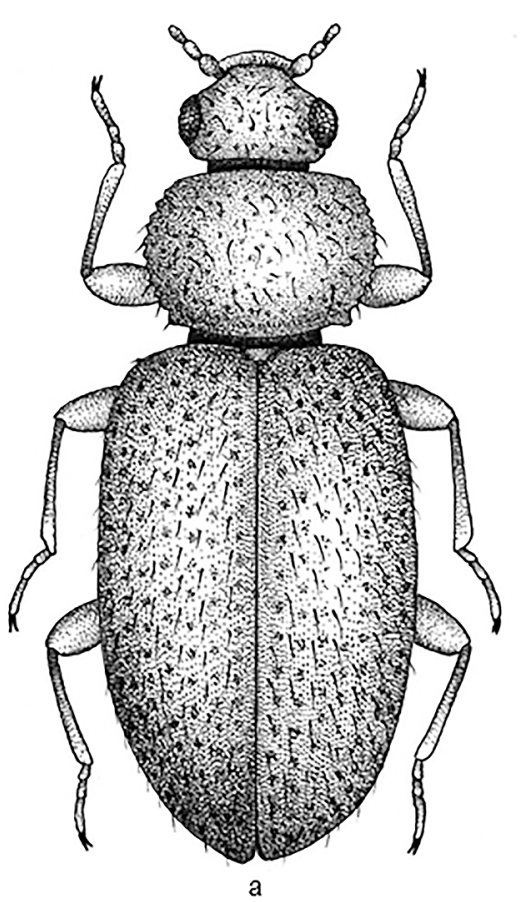

\subsection{Corticaria franzi Dajoz (Fig. 9)}

Corticaria franzi Dajoz 1969: 96

Type material examined. Holotype (+): Spain, Sierra Guadarrama, El Escorial (leg. H. Franz) (NMW). Paratype (1 ex): Spain, Madrid, Valle de la Fuenfría, Sierra de Guadarrama (leg. H. Franz) (MNHN).

Other specimens examined. Spain. Madrid, without date, 1ex (leg. G. A. del Real) (MNCN). Madrid, El Escorial, without date, 4 exx (MNCN). Madrid, El Escorial, without date, $1 \mathrm{ex}$ (leg. H. Franz) (MNHN). Madrid, Hoyo de Manzanares, 21.X.1951, 1 ex (leg. E. Humbert) (MNCN). Madrid, Robledo de Chavela, 14.X. 1973, 21 exx (leg. S. Pérez). Madrid, Lozoya, Arroyo del Villar, 14.VI.1980, 1 ex (leg. P. Gamarra) (USC).

Morphology. Body. Length 1.6-1.7 mm, oval and convex (Fig. 9a). Yellowish-brown. Yellow pubescence, long $(\mathrm{L}=37-53 \mu \mathrm{m})$ and raised. Metathoracic wings absent. Head little transverse $(\mathrm{WL}=1.3-1.4)$. Puncturation fairly thick $(\varnothing=$ 22-29 $\mu \mathrm{m})$.

Head. Hemispherical eyes, large $(\mathrm{L}=0.114$ $\mathrm{mm})$ and little prominent $(\mathrm{E}=0.7)$. Ocular facets smaller $(\varnothing=18-19 \mu \mathrm{m})$ than punctures of head.

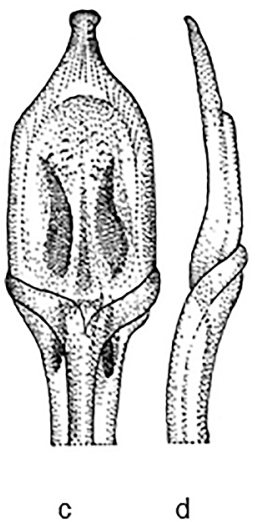

Fig. 9. Corticaria franzi Dajoz. - a. Dorsal habitus. - b. Antenna. - c. Aedeagus, dorsal view. $-\mathrm{d}$. Aedeagus, lateral view.

Temples little visible with a pair of setae directed forward. Antennae (Fig. 9b) thin, pubescent and short $(\mathrm{L}=0.509-0.511 \mathrm{~mm})$, not reaching base of pronotum. Antennomere I strongly dilated and elongated; antennomere II, 1.7 times longer than broad; III as long as II, but slightly thinner; IV to VIII longer than broad, becoming gradually shorter; VIII transverse; IX subconical; X shorter than IX; XI elongated and obliquely truncated in apex.

Pronotum (Fig. 9a). Moderately transverse $(\mathrm{WL}=1.5-1.6)$; narrower at base than at apex. Lateral margins ciliated and dentate, with a larger tooth near base. Prebasal pit absent. Puncturation shallow; punctures separated by a distance shorter than their diameter $(\varnothing=13-21 \mu \mathrm{m})$. Elytra together 2.5 times longer than pronotum and 1.5 times longer than broad. Elytral margin not visible dorsally. Male protibiae with a small spine in internal apical area.

Abdomen. Ventrite 1 shorter than next three together. Distal margin of ventrite 5 slightly concave.

Aedeagus (Fig. 9c, d). With apex of tegmen slightly spatulate and asymmetric. Sharp in lateral view. Internal sac with four large rods and in middle of them other small ones. 
Distribution. Central Spain (Dajoz 1969, Johnson 2007, Rücker 2013a).

Biology. The examined specimens have been collected from remains of Juniperus sp.

\subsection{Corticaria fulvoides C. Johnson (Fig. 10)}

\section{Corticaria fulvoides C. Johnson 1989: 82}

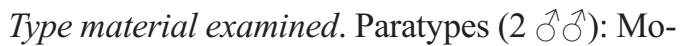
rocco, Haut-Atlas, Oukaimeden, 2,500 m, 28.IV. 1960 (leg. C. Besuchet) (MMCJ).

Morphology. Body. Length 1.6-1.8 mm, moderately convex (Fig. 10a). Entirely yellowish-brown, antennae a little paler. Pubescence double; interstitial hairs rather sparse, semi-erect, curved, very outstanding and long. Metathoracic wings absent.

Head. A little narrower than pronotum, width $0.35-0.38$; reticulation well marked, puncturation rather spaced, somewhat fine and shallow. Temples well developed about half-length of eyes, only feebly widened and somewhat obtusely angulate behind; eyes only slightly wider than temples. Antennae (Fig. 10b) moderately long, length $0.58-0.59 \mathrm{~mm}$, club abrupt but rather short.

Pronotum. Rather small, broadest slightly in front although rarely across middle, breadth
$0.48-0.51 \mathrm{~mm} ; 1.23-1.30$ times as broad as long; sides moderately rounded, with 8-9 moderate sized crenulations and swept back lateral pubescence, hardly projecting; surface shining, alutaceous microsculpture very distinct, nearly a diameter apart on disc; pot median depression profound, lateral impressions absent; hind angles each with a long outstanding seta. Elytra together somewhat short oval, 2.69-2.79 times as long as pronotum, 1.56-1.62 times as long as broad; slightly more convex and wider than pronotum, breadth $0.64-0.72 \mathrm{~mm}$; sides conspicuously curved, distinctly serrate basally. Prosternum with pubescent transverse fovea antero-lateral to procoxae. Mesocoxae very narrowly separated by acuminate process. Metasternum short, 0.67 times length of ventrite 1 medially, hind margin of intercoxal process notched medially. Legs moderately long. Male: pro and mesotibiae with a very small tooth on inner edge at apex; protarsi with basal segment only slightly dilated.

Abdomen. Ventrite 1 somewhat finely and sparsely punctured. Ventrite 5 broadly impressed medially.

Aedeagus (Fig. 10c, d). With pointed end.

Distribution. SW Morocco (Johnson 2007, Rücker 2013a).

Biology. Unknown.
Fig. 10. Corticaria fulvoides Johnson. - a. Dorsal habitus. - b. Antenna. - c. Aedeagus, dorsal view. - d. Aedeagus, lateral view.

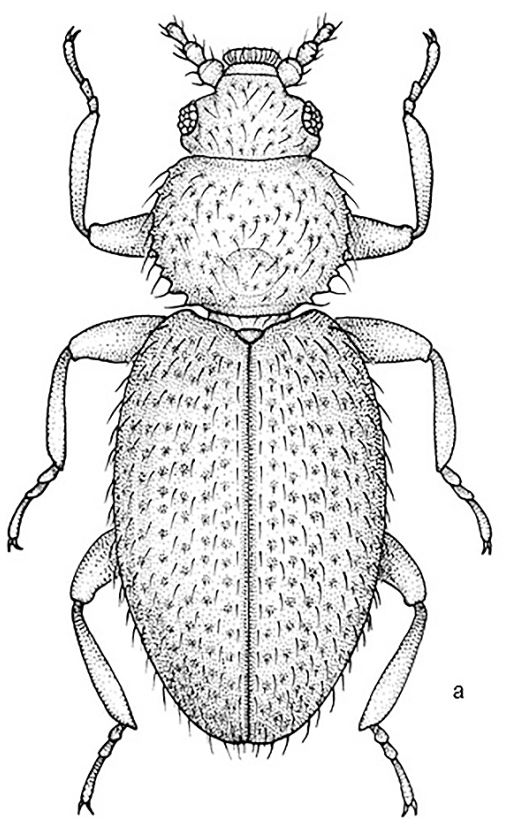

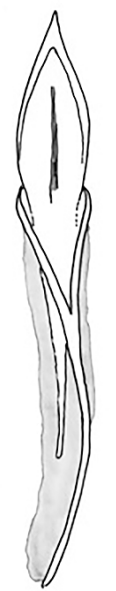

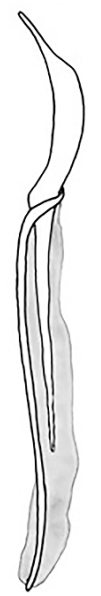

c d 


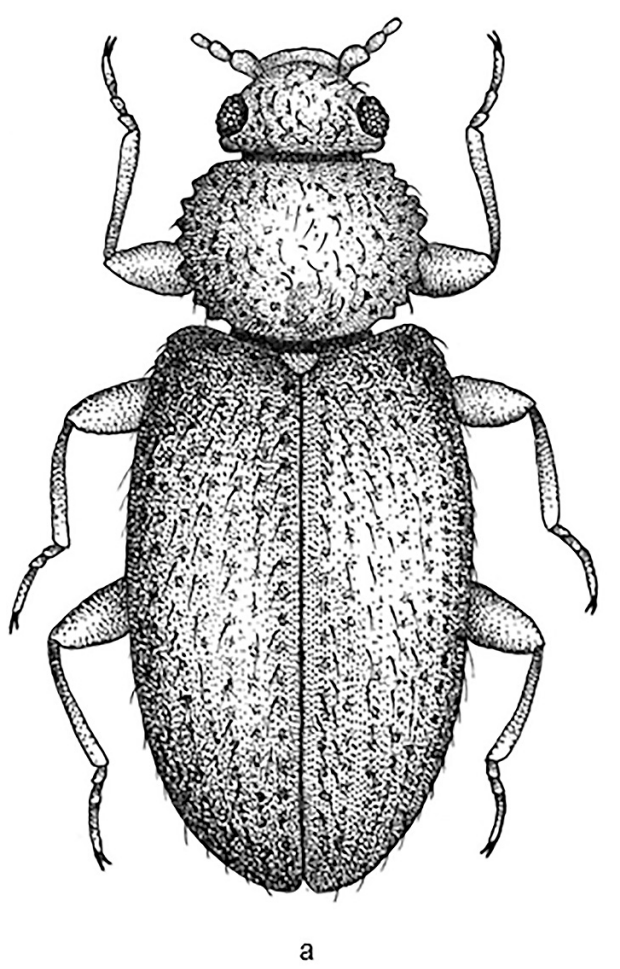

3.11. Corticaria johnsoni Mariño, López \& Otero (Fig. 11)

Corticaria johnsonii Mariño, López \& Otero 2007: 24

Type material examined. Holotype (ठ): Spain, Ávila, Sierra del Valle, Puerto de Casillas, 1100 m. 1.XI.1975. (leg. L. Gil) (USC).

Morphology. Body. Length $1.9 \mathrm{~mm}$, oval and convex (Fig. 11a). Colour yellowish brown. Elytra with 8 striae made up of rows of weakly marked punctures. Long white pubescence $(\mathrm{L}=$ 75-90 $\mu \mathrm{m})$. Wingless.

Head. Transverse (WL $\geq 2.0$ ), semicircular. Eyes large $(\mathrm{L}=0.125 \mathrm{~mm})$ and scarcely prominent $(\mathrm{E}=0.4-0.5)$. Eyebrows protruding $(\mathrm{L}=$ $24.9 \mu \mathrm{m})$. Antennae (Fig. 11b) with 11 antennomeres, almost reaching posterior margin of pronotum $(\mathrm{L}=0.600 \mathrm{~mm})$. Antennomere I, 1.5 times longer than II; II oval, 2 times longer than wide, 0.3-0.4 times longer than III; III almost 2 times longer than wide; IV, V and VI 0.5 times longer than wide; VII and VIII sub-square; IX, X and XI

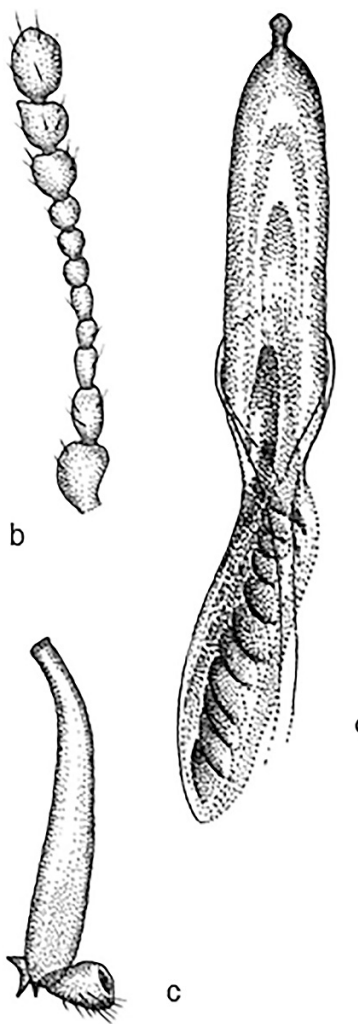

Fig. 11. Corticaria johnsoni Mariño, López \& Otero. - a. Dorsal habitus. $-b$. Antenna. - c. Protibiae. - d. Aedeagus, dorsal view.

forming club, IX and XI longer than wide, $\mathrm{X}$ transverse. Two pits below clypeus and forehead, like those described in C. ruckeri, with openings conspicuous in frontal view, and reaching half depth of eyes. These invaginations probably have hygrosensory function.

Pronotum. Concave (Fig. 11a), little transverse $(\mathrm{WL}=1.3-1.4)$, with width maximal in anterior third. Anterior margin longer than posterior margin. Basal pit present, but faintly marked. Lateral margins forming a not pointed angle at point of maximum pronotum width, with 8-9 conspicuous teeth. Scutellar shield flat, transverse. Elytra together oval, 1.5 times longer than wide, 3 times longer and 0.3 times wider than pronotum. Elytrum width maximal in medial part. Lateral margin well marked anteriorly, with conspicuous shoulders $(\mathrm{L}=30 \mu \mathrm{m})$. Protibiae (Fig. 11c) with two spines in their internal apical angles.

Aedeagus (Fig. 11d). Elongated to final quarter of its length, in which with a triangular lancelike shape. Gradually narrowing anteriorly, with an abrupt curve at anterior tip, giving rise to a small petiole $(\mathrm{L}=38 \mu \mathrm{m})$ terminating in a semi- 
circular dilatation. Internal sac with a dorsal row of 10 long slightly curved rods $(\mathrm{L}=60-100 \mu \mathrm{m})$.

Female. Not known.

Distribution. Central Spain (Mariño et al. 2007, Rücker 2013a).

Biology. The specimen was collected from oak foliage.

\subsection{Corticaria lucasi Otero, López \& Rücker}

(Fig. 12)

Corticaria lucasi Otero, López \& Rücker 2013: 238

Type material examined. Holotype ( $\left.{ }^{(}\right)$: Spain, Murcia, Lorca, Pantano de Valdeinfierno [37 $48^{\prime}$ 62" N, 1ㅇ 58’ 52” W; 25.V.1979] (leg. J. J. Presa) (USC).

Paratypes: $1 \hat{\jmath}$ (WRC); 1 क (UMC); $2 \hat{\jmath} \sigma^{\lambda}$ (USC): same locality and data as Holotype.

Morphology. Body. Length 1.6-1.8 mm, short, oval and convex (Fig. 12a). Testaceous yellow. Yellowish pubescence, long $(\mathrm{L}=64-68 \mu \mathrm{m})$ and erect in interstriae, but shorter and recumbent in striae. Metathoracic wings absent.
Head. Moderately transverse $(\mathrm{WL}=1.6)$, slightly punctuated and reticulated. Eyes hemispheric, large $(\mathrm{L}=0.109 \mathrm{~mm})$, and scarcely protuberant $(\mathrm{E}=0.8)$. Ocular facets $(\varnothing=8-13 \mu \mathrm{m})$ smaller than punctures of head $(\varnothing=16-17 \mu \mathrm{m})$. Angular temples, equal to $1 / 3$ of length of eye, with setae oriented towards front. Antennae (Fig. 12b) narrow, pubescent and long $(\mathrm{L}=0.607 \mathrm{~mm})$, reaching posterior edge of pronotum. Antennomere III to VI longer than broad; VIII ovoid and as long as broad. Antennal club elongated. IX antennomere as long as broad.

Pronotum (Fig. 12a). Very convex, heartshaped and moderately transverse (WL $=1.6-$ 1.7); a little narrower in base of apex; at widest in anterior third. Anterior margin straight. Anterior angles rounded, posterior angles obtuse. Lateral margin rounded, ciliated, with a strong denticulation, more evident in posterior half, ending in two large teeth. Prebasal pit rounded and moderately impressed. Punctuation strong and separated; punctures separated by a distance longer or equal to their diameter $(\varnothing=19-22 \mu \mathrm{m})$ (Fig. 12c). Scutellar shield transverse and flat. Elytra
Fig. 12. Corticaria lucasi Otero, López \& Rücker. - a. Dorsal habitus. - b. Antenna. - c. Puncturation. - d. Aedeagus, dorsal view. - e. Aedeagus, lateral view.
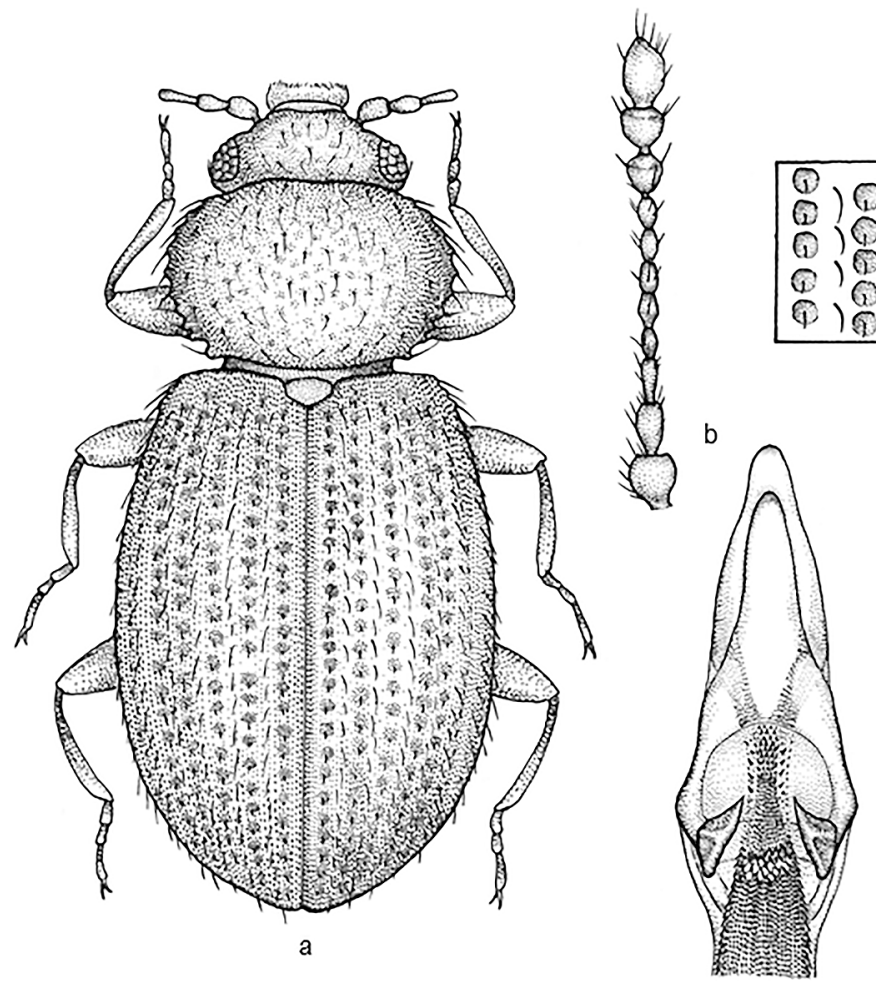

d
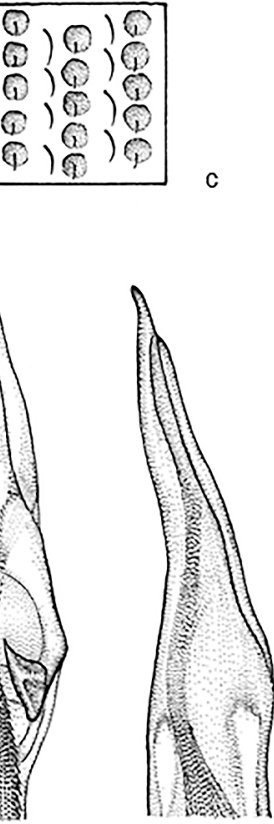

e 


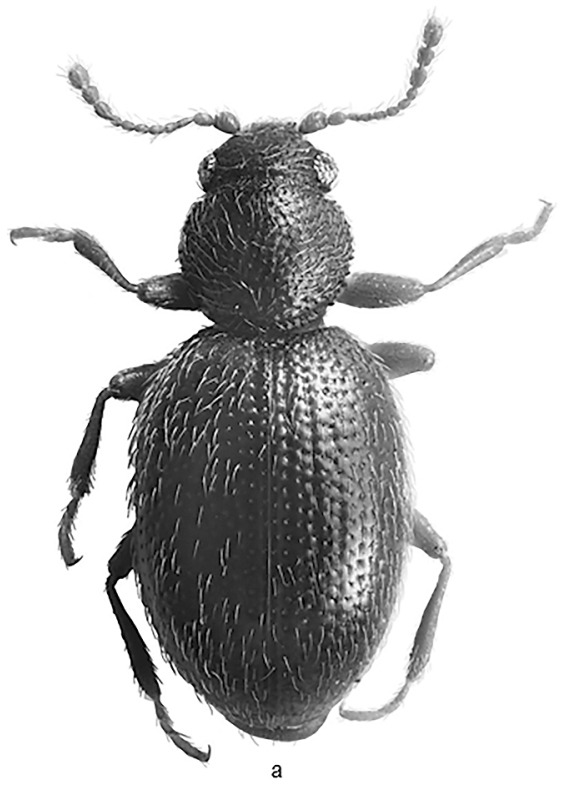

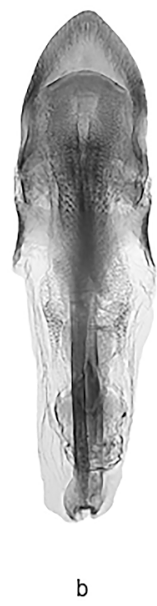

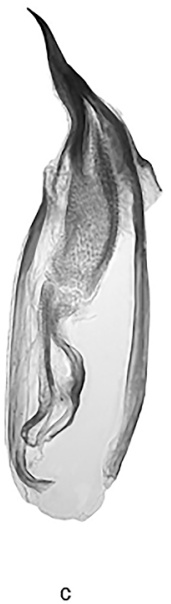

Fig. 13. Corticaria meybohmi Reike. - a. Dorsal habitus. $-b$. Aedeagus, dorsal view. - c. Aedeagus, lateral view (after Reike 2015). together very convex, 1.3 times broader and 3 times longer than pronotum. Lateral border crenulated in humeral angle. Striae strongly punctuated, with thick and almost contiguous punctures. Without precoxal pits in prosternum. Anterior tibiae straight in their internal margin, with a terminal spine and cut obliquely in apex of their external margin. First segment of tarsi dilated and ciliated underneath.

Abdomen. Ventrite 1 twice as long as prosternum, with a weak and separated puncturation. Ventrite 5 slightly concave in its distal margin.

Aedeagus (Fig. 12d). Length $0.370 \mathrm{~mm}$; apex rounded and symmetrical, making an obtuse angle in lateral view (Fig. 12e). Internal sac with two large conical sclerotized rods $(\mathrm{L}=63 \mu \mathrm{m})$, base with numerous smaller ones in shape of a needle.

Female. Same external morphological characters as male.

Distribution. SE Spain (Otero et al. 2013).

Biology. In vegetal products in process of decomposition.

\subsection{Corticaria meybohmi Reike (Fig. 13)}

\section{Corticaria meybohmi Reike 2015: 1}

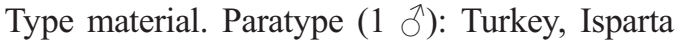
Kovada Gölu, $890 \mathrm{~m}$ ü. NN, Platanenstreu, 37³7'42’N, 3051'39’E, 15.IV.2008 (leg.
Meybohm and Brachat) (coll. W. Rücker).

Morphology. Body. Length $1.82 \mathrm{~mm}$, long, oval with lateral margins strongly arcuate (Fig. 13a). Reddish brown; tarsi and antennae lighter. Pubescence yellowish, long and elevated. Metathoracic wings absent. Head. Highly transverse $(\mathrm{WL}=2.6)$. Eyes $(\mathrm{L}=100 \mu \mathrm{m})$ coarsely faceted and slightly protuberant. Eye facets as large as head punctures. Very short temples $(\mathrm{L}=$ $0.019 \mathrm{~mm})$. Antennae thin, pubescent and long ( $\mathrm{L}$ $=0.625 \mathrm{~mm}$ ). Antennomere I large and spherical; antennomere II narrower than I but twice as wide as III; antennomere III long and narrow; IV and VII longer than wide; VIII oval, as long as wide. Antennomere IX longer than wide; $\mathrm{X}$ wider and shorter than IX; XI longer, truncated in apex.

Pronotum (Fig. 13a). Convex, cordiform and slightly transverse $(\mathrm{WL}=1.2)$, narrower in base than in apex; reaching its maximum width in middle front. Anterior margin straight. Anterior angles rounded and strongly obtuse. Lateral margins rounded and slightly denticulate. Without basal fossula. Puncturation similar to that of head. Scutellar shield transverse and flat. Elytra together strongly convex; 1.3 times longer than wide. Lateral rim slightly marked. Punctures separated by a greater distance than their diameter. First tarsomere dilated and pubescent below. Internal margin of tibiae slightly arcuate and with terminal spines. 
Abdomen. Ventrite 1 almost as long as next three together. Ventrite 5 flat, with several rows of setae on apex of its external margin.

Aedeagus (Fig. 13b). Triangular with apex rounded. Sinuate in lateral view (Fig. 13c). Apex forms an obtuse angle in its apical section. Internal sac with numerous small denticles.

Distribution. Turkey, Isparta (Reike 2015).

Biology. Collected in banana plantations (Reike 2015).

\subsection{Corticaria pinicola C.N.F. Brisout de Barneville (Fig. 14)}

Corticaria pinicola C.N.F. Brisout de Barneville 1866: 370

Corticaria rufescens Reitter 1875: 413, 420

Type material examined. Lectotype (ठ): Spain. Escorial, pinicola $\mathrm{Ch}$. Bris., Mules Cent Reitt. (MNHN) (Rücker 2013a).

Paralectotypes 2 우: Same data (MNHN) (Rücker 2013b).

Other specimens examined. Spain. Ávila, Navarredonda de Gredos, 18.III.1990, 3 exx (leg. M. Costas). Ávila, Piedralavés, 3.V.1975, 1 సَ (leg. V. Monserrat). Ávila, Puerto de Malagón, 31.III.1976, 1 q (leg. R. Outerelo). Asturias, Cangas de Onís, 3 exx (leg. H. Paganetti) (MNHN). Madrid, Fuente de los Geólogos, 2.X.1974, 1 q (leg. R. Outerelo). Madrid, Aranjuez, 1 ex (leg. E. Reitter) (MNHN). Madrid, Sa Gredos, Puerto de Casillas, 1.X.1975, 2 $\widehat{\partial}$ (leg. L. Gil). Madrid, Sierra de Guadarrama, 3 exx (leg. H. Franz) (MNHN). Madrid, Camorritos, 7.IX.1975, 1 ㅇ (leg. R. Outerelo).

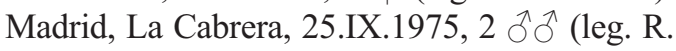
Outerelo). Madrid, Puerto de Navacerrada, 10.X.1975, 1 \& (leg. R. Outerelo). Madrid, La Cabrera, 21.X.1975, 2 §ิð (leg. R. Outerelo). Madrid, El Escorial, La Herrería, 24.X.1975, 1 ภ (leg. R. Outerelo). Madrid, La Quebrada, 14.I.1976, 1 đ̋ (leg. R. Outerelo). Madrid, Líjar,

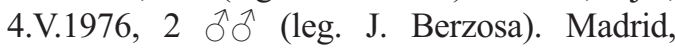
Miraflores de la Sierra, 12.V.1977, 1 đo and 1 ㅇ (leg. R. Outerelo). Madrid, Rocafría, 27.VIII.1977, 1 \& (leg. R. Outerelo). Madrid, El

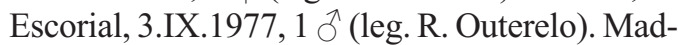
rid, Cercedilla, 4.IX.1977, 2 우 (leg. R. Outerelo) (USC).

Morphology. Body. Length 1.4-2.0 mm, oval, elongated and convex (Fig. 14a). Reddish-
Fig. 14. Corticaria pinicola Brisout de Barneville. - a. Dorsal habitus. - b. Antenna. - c. Aedeagus, dorsal view. - d. Aedeagus, lateral view. - e. Internal sac.
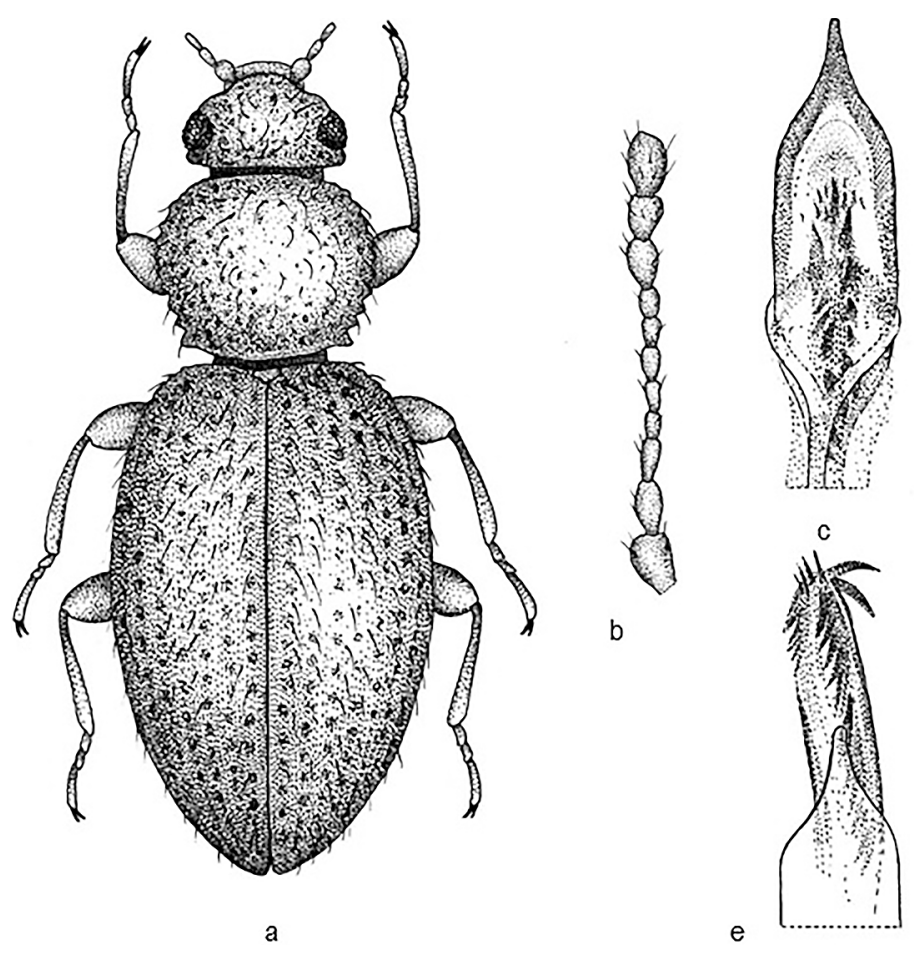


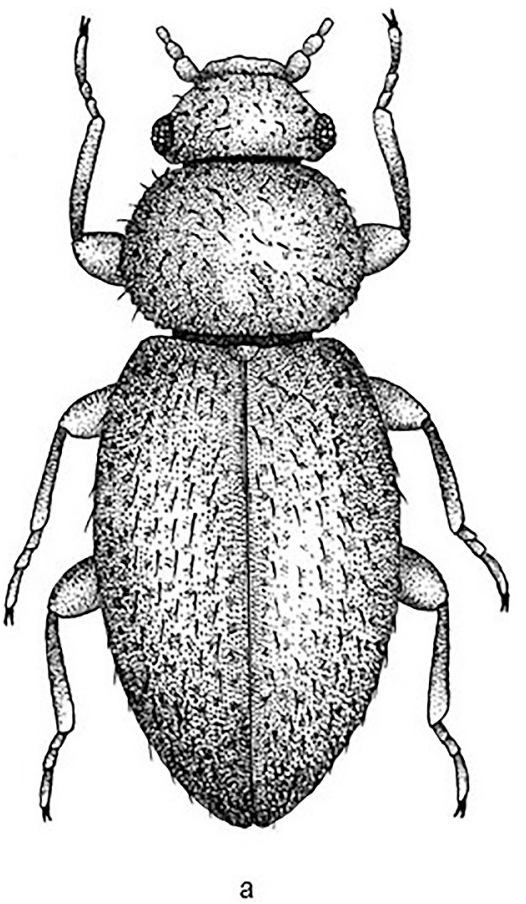

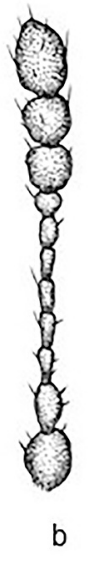

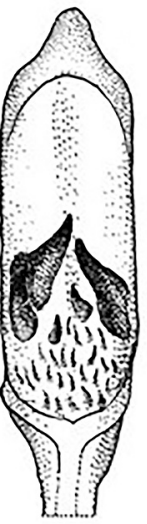

d

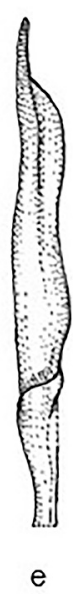

Fig. 15. Corticaria punctata Dajoz. - a. Dorsal habitus. - b. Antenna. - c. Sternite 5. - d. Aedeagus, dorsal view. - e. Aedeagus, lateral view. brown. Long yellowish pubescence $(\mathrm{L}=63-71$ $\mu \mathrm{m})$. Metathoracic wings absent.

Head. Moderately transverse (WL $=1.4-1.5)$. Clypeus and labrum quite outstanding, both located on same plane. Two very deep pits under clypeus; their length half of length of eye. Eyes hemispherical, small $(\mathrm{L}=95-105 \mu \mathrm{m})$ and prominent $(E=1.0)$. Ocular facets smaller $(\varnothing=6-9$ $\mu \mathrm{m})$ than head punctures $(\varnothing=12-14 \mu \mathrm{m})$. Temples well-marked, short and $1 / 3$ as long as length of eye. Antennae (Fig. 14b) thin, pubescent and short $(\mathrm{L}=0.616 \mathrm{~mm})$, not reaching base of pronotum. Antennomere I dilated and semispherical; II as long as I, but narrower; III shorter and narrower than II, but longer than following ones; IV to VII longer than broad, progressively shorter and broader; VIII squared, almost as long as broad; IX dilated, longer than broad; $\mathrm{X}$ as broad as IX but shorter; XI obliquely truncated in apex, forming an asymmetric shape.

Pronotum (Fig. 14a). Convex and little transverse $(\mathrm{WL}=1.2-1.3)$, broadest in middle part. Lateral margins rounded and strongly crenulated; denticles slightly larger in posterior part. No basal pit or little distinct. Puncturation $(\varnothing=23-28 \mu \mathrm{m})$ visible and disperse; punctures separated by a distance longer than their diameter. Pronotal pubes- cence long and raised, projecting from denticle tips, especially visible. Scutellar shield transverse and flat. Elytra together oval and elongated, almost three times as long as pronotum. Lateral margin well-marked only in anterior third, humeral calus barely distinct. Pubescence very long $(\mathrm{L}=60-97 \mu \mathrm{m})$ and raised. Strial puncturation very strong $(\varnothing=35-40 \mu \mathrm{m})$. Metasternum shorter than ventrite 1 . Anterior and medial tibiae with spines in internal apical angle.

Aedeagus (Fig. 14c, d). Length 0.450-0.470 $\mathrm{mm}$, asymmetric, sharp and truncated in apex. Internal sac (Fig. 14e) with three rods, two lateral well-developed $(\mathrm{L}=95-105 \mu \mathrm{m})$ and a central third one barely distinct.

Distribution. Central Spain (Johnson 2007, Rücker 2013a).

Biology. Collected in pine litter (Belon 1897).

\subsection{Corticaria punctata Dajoz (Fig. 15)}

\section{Corticaria punctata Dajoz 1970: 261}

Type material examined. Holotype (q): Spain, Alicante, El Peñón (leg. H. Franz) (NMW).

Other specimens examined. Spain. Barcelona, Sarriá, 10.X.1919, 1 q (leg. F. Español) (MZB). 
Morphology. Body. Length 1.6-1.7 mm, oval, elongated and convex (Fig. 15a). Light testaceous yellow. Yellowish-white pubescence of average length and little raised. Barely coloured eyes. Metathoracic wings absent.

Head. Transverse (WL $=1.8-1.9)$ with deep and compact puncturation, almost adjacent. Small eyes made up of few thick facets ( 5 along eye length). Temples short, half eye length. Antennae long $(\mathrm{L}=0.535 \mathrm{~mm})$ (Fig. 15b). Antennomere I dilated and spherical; II narrower, however, almost as long as I, II to VII longer than broad, each one progressively shorter and broader than latter, VIII completely transverse, IX and X dilated, subconical and transverse; XI longer than previous ones and truncated in apex.

Pronotum (Fig. 15a). Little transverse (WL $=$ 1.4), oval, little convex with a basal pit slightly marked. Lateral margins curved with strong denticulation along almost its whole length, largest teeth in posterior half. Scutellar shield small and transverse. Elytra together elongated (1.61.7 times longer than broad), convex, lateral margin only marked in most anterior area. Striae with light and dispersed puncturation along their whole length. Interstriae not punctured, flat in their posterior part and slightly carenate at base. Two large and transverse pits in front of anterior coxa. Flat metasternum as long as two thirds of ventrite 1 . Male anterior tibiae widened in their distal third and obliquely serrated in their internal apical angle.

Abdomen. Ventrite 5 (Fig. 15c) narrowed in medial area, slightly prolonged backwards. This extension is concave, spoon-shaped, with a stripe of small setae in posterior margin. Aedeagus (Fig. $15 \mathrm{~d}, \mathrm{e})$. Strongly narrowed right before apex in dorsal view, whereas with sharp profile. Internal sac with three large rods and others smaller and numerous at base.

Distribution. E Spain (Johnson 2007, Rücker 2013a)

Biology. Unknown.

\subsection{Corticaria rueckeri Otero, Mariño \&} López (Fig. 16)

Corticaria rueckeri Otero, Mariño \& López 2006: 374
Type material examined. Holotype (ð): Spain. Madrid, Puerto de la Morcuera, 21.X.1976 (leg. R. Outerelo) (USC).

Paratypes. 1 đ: Spain. Madrid, Valdemartin, 11.XI.1974 (leg. R. Outerelo) (MNCN). 1 ㅇ: Madrid, Navacerrada, 18.V.1976 (leg. R. Outerelo) (MNCN). 1 o : Madrid, Arroyo Sestil, 21. X.1976. 1 o: Madrid, Arroyo Sestil, 24.III.1977 (leg. R. Outerelo) (USC). 1 ex: Madrid, Barranco de Navacerrada, 5.IV.1980 (leg. R. Outerelo) (USC). 1 $\lesssim$ : Madrid, Puerto de la Morcuera, 21.X.1976 (leg. R. Outerelo) (MNHG). 1 đ: Madrid, Puerto de Malagón, 24.II.1976 (leg. R. Outerelo) (USC). 1 § : Madrid, El Escorial, 3.IX.1977 (leg. R. Outerelo) (MZLU). 1 స: Segovia, El Espinar, Garganta de río Moros, 26.V.1977 (leg. R. Outerelo) (NMW). 1 ऽ̊: Segovia, El Espinar, Monte El Petril, 25.VI.1977 (leg. R. Outerelo) (MNHUB). 1 : Segovia, Puerto de los Leones, 2.X.1975 (leg. R. Outerelo) (WR). 1 đ̃: Madrid, Puerto de la Morcuera, 21.X.1976 (leg. R. Outerelo) (USC). 1 : Segovia, Puente de la Cantina, 20.V.1976 (leg. R. Outerelo) (USC).

Morphology. Body. Length 1.7-1.8 mm, oval and convex (Fig. 16a). Colour yellowish brown to straw-coloured. Puncturation present over entire body $(\varnothing=20 \mu \mathrm{m})$, with a small orifice of unknown function at base of each puncture. Eight patent striae on each elytron. Long white pubescence $(\mathrm{L}=60-70 \mu \mathrm{m})$. Wingless.

Head. Transverse $(\mathrm{WL}=1.7)$ and slightly pointed towards apex. Pitting somewhat deeper than in rest of body. Eyes medium-sized ( $\mathrm{L}=80$ $\mu \mathrm{m})$ and scarcely prominent $(\mathrm{E}=0.63)$, with 25 ocelli $(\varnothing=18-20 \mu \mathrm{m})$. Temples visible, somewhat shorter $(\mathrm{L}=63-65 \mu \mathrm{m})$ than eyes. Antennae (Fig. 16b) with 11 antennomeres, extending to posterior third of pronotum $(\mathrm{L}=500 \mu \mathrm{m})$. Antennomere I, 1.5 times wider than II; II oval, 2 times wider than III; III 3 times longer than wide; IV, V and VI almost 2 times longer than wide; VII only slightly longer than wide; VIII subsquare; IX, $\mathrm{X}$ and XI longer than wide, forming well-developed club. Between eyes and mouth, and below clypeus and frons, with two conspicuous pits (Fig. 16c), blind-ended and broadly conical, converging to center of head, and reaching half depth of eyes; openings of these pits visible in frontal view $(\varnothing=30-35 \mu \mathrm{m})$, slightly inclined with re- 


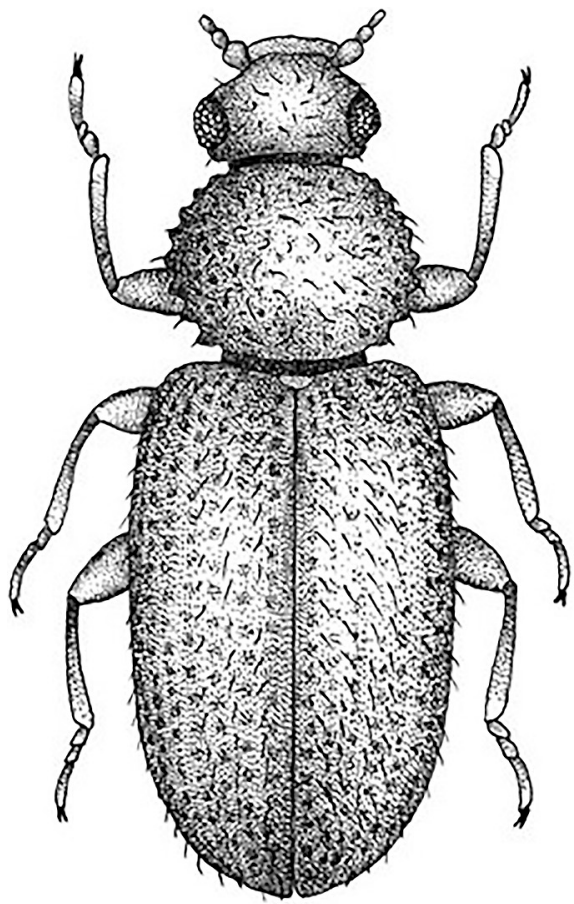

a

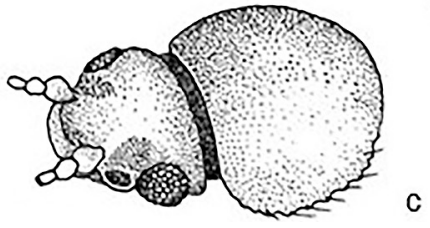

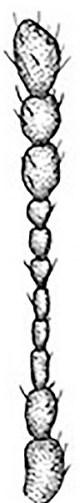

b

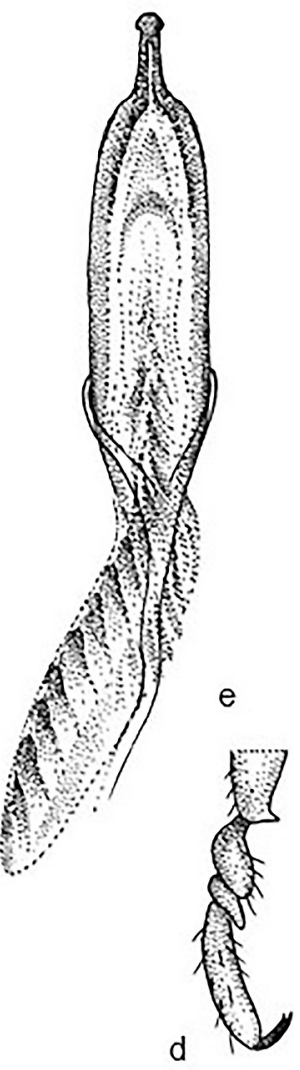

Fig. 16. Corticaria rueckeri Otero, Mariño \& López. - a. Dorsal habitus. - b. Antenna. - c. Cephalic pits. - d. Protibial ápex. - e.

Aedeagus, dorsal view. spect to sagittal plane. Function of cephalic pits not known, but may be related to hygrosensation.

Pronotum (Fig. 16a). Convex, moderately transverse $(\mathrm{WL}=1.4-1.5)$. Anterior and posterior margins of similar length, pronotum width maximal in median part. Basal pit faintly marked. Margins rounded with 6-8 well-marked denticles, posterior denticle largest. Scutellar shield transverse, flat. Elytra together oval, elongated, 1.6 times longer than wide. Elytron length 2.63.0 times pronotum length and 0.5 times longer than maximum pronotum width. Lateral margin faintly marked, except in its anterior part. Tibiae with spine on internal apical angle (Fig. 16d), longer on protibiae, more conspicuous in males.

Abdomen. Ventrite 1 one third length of metasternite.

Aedeagus (Fig. 16e). Elongated and pointed to final quarter of its length, in which it acquires a triangular lance-like shape. An apex anteriorly, one third of total length of aedeagus, with an ar- rowhead shape. In lateral view, aedeagus as a descending curve, accentuated at anterior tip. Internal sac (Fig. 16e) with one row of sclerotized rods, and a second row of smaller spines running parallel with first row.

Distribution. Central Spain (Otero et al. 2006, Rücker 2013a).

Biology. Unknown.

\subsection{Corticaria suspecta C. Johnson (Fig. 17)}

\section{Corticaria suspecta C. Johnson 1989: 84}

Type material examined. Paratype (ठへ): Morocco, Tizi-n-Test, 30.IV.1960 (leg. C. Besuchet) (MMCJ).

Morphology. Body. Length 1.6-1.8 mm, moderately convex (Fig. 17a). Entirely yellowish brown. Pubescence double $(\mathrm{L}=40-74 \mu \mathrm{m})$; interstitial hairs closed, semi-erect and curved. Metathoracic wings absent. 
Fig. 17. Corticaria suspecta Johnson. - a. Dorsal habitus. $-b$. Antenna. - c. Aedeagus, dorsal view. - d. Aedeagus, lateral view.

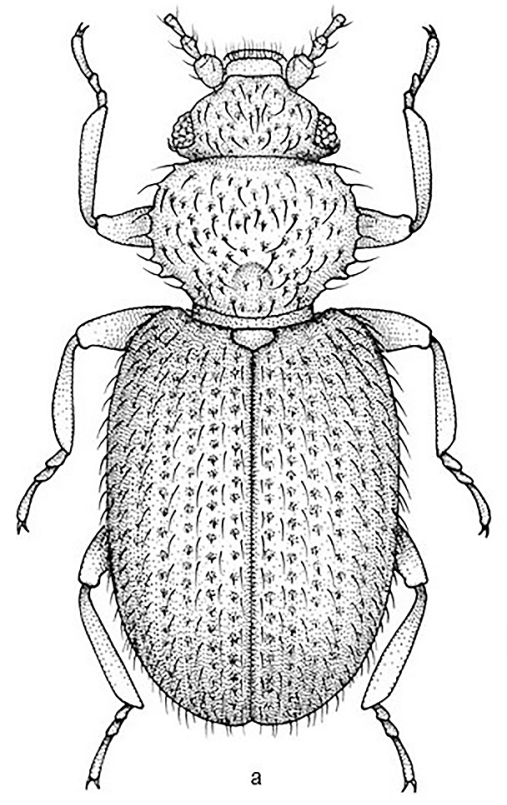

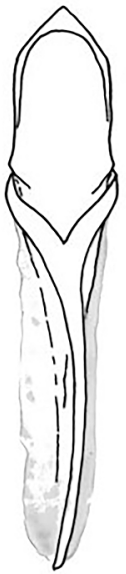

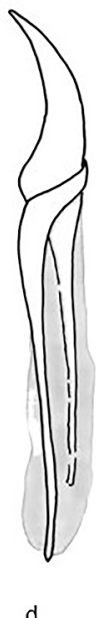

C d
Head. Narrower than pronotum, moderately transverse (WL $=1.5-1.6)$. Reticulation well marked, puncturation $(\varnothing=12-18 \mu \mathrm{m})$ rather spaced, somewhat fine and shallow. Eyes medium-sized $(\mathrm{L}=85-90 \mu \mathrm{m})$ and scarcely prominent $(E=0.7)$. Temples well developed, over half of length of eyes, not widened behind, extreme hind angles slightly rounded off; eyes only slightly wider than temples. Antennae (Fig. 17b) moderately long $(\mathrm{L}=0.38-0.43 \mathrm{~mm})$, club abrupt but rather short; antennomere VIII broader than long, IX barely, X distinctly, broader than long.

Pronotum (Fig. 17a). Moderately transverse $(\mathrm{WL}=1.6-1.7)$. Sides moderately rounded, with at least eight rather weak crenulations, and swept back lateral pubescence, hardly projecting; surface rather shining, alutaceous microsculpture fine but distinct, punctures moderate in size but not deep, mostly about a diameter apart on disc; post median depression weak, lateral impressions absent. Elytra together somewhat short oval, 2.82.9 times as long as pronotum, 1.5-1.6 times as long as broad; slightly more convex and clearly wider than pronotum, breadth $0.68-0.72 \mathrm{~mm}$; sides rather moderately curved, distinctly serrate basally. Prosternum with pubescent transverse fovea antero-lateral to procoxae. Mesocoxae very narrowly separated by acuminate process. Metasternum short, 0.50 times length of ventrite
1 medially. Legs moderately long. Male: pro and mesotibiae with a very small tooth on inner edge at apex; protarsi with basal segment only slightly dilated.

Abdomen. Ventrite 1 somewhat finely and sparsely punctured. Ventrite 5 broadly impressed medially.

Aedeagus (Fig. 17c, d). With triangular apex.

Distribution. SW Morocco (Johnson 2007, Rücker 2013a).

Biology. Unknown.

\subsection{Corticaria sylvicola C. N. F. Brisout de Barneville (Fig. 18)}

Corticaria sylvicola C. N. F. Brisout de Barneville 1863: 72

Corticaria pinguis Aubé 1866: 162

Type material. Not examined.

Other specimens examined. France, Pyrénèes Orientales, Madeloc, Banyuls, 7.IV.1963, 1 ○े. France, Pyrénèes Orientales, Port-Vendres, 12.IV.1965, 1 ○ (leg. G. Tempère) (USC). Spain. Asturias, Prades, 2 exx (leg. V. Xambeu) (MNHN). Tarragona, Cadaqués, 14 exx (leg. H. Franz) (MNHN). Teruel, Sierra de Albarracín (WRC).

Morphology. Body. Length 1.5-1.75 mm, 


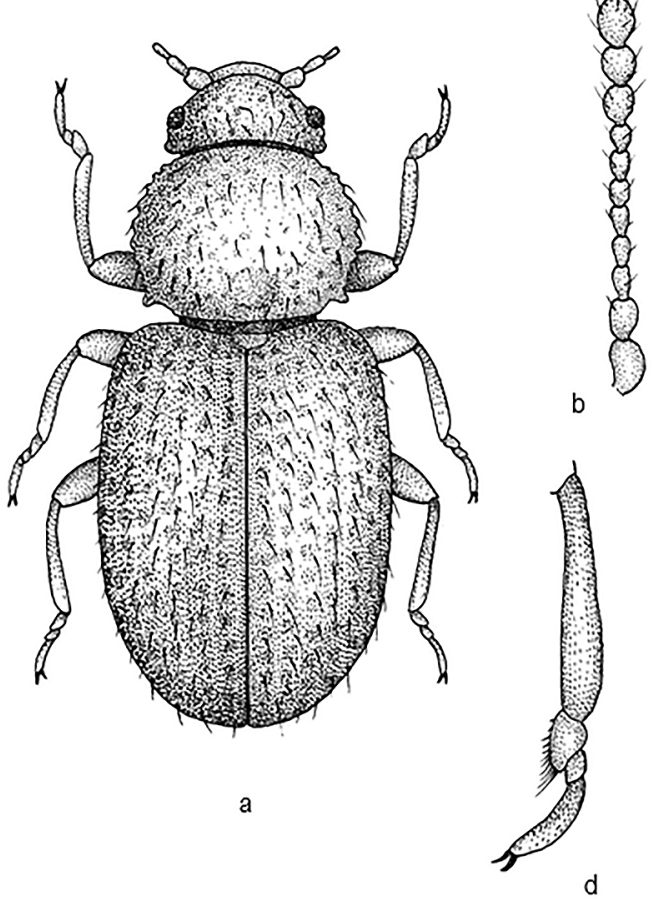

short, oval and very convex (Fig. 18a). Ferruginous brown. Yellowish pubescence, long, relatively raised and thick. Metathoracic wings absent.

Head. Very transverse (WL $=2.1-2.2)$ with cephalic thick puncturation. Pubescence very long $(\mathrm{L}=50-70 \mu \mathrm{m})$ and raised. Eyes small $(\mathrm{L}=$ $50-60 \mu \mathrm{m})$, scarcely protuberant $(\mathrm{E}=0.45)$ made up of a few thick ommatidia. Angular temples as long as ocular one. Antennae long (Fig. 18b) $(\mathrm{L}=$ $0.550 \mathrm{~mm}$ ), almost reaching posterior margin of pronotum. All antennomeres longer than broad, however, VIII and antenonmeres of club tend to a slightly more transverse shape.

Pronotum (Fig. 18a). Moderately transverse $(\mathrm{WL}=1.5-1.6)$, largest width in its medial part, almost as wide as elytra. Lateral margins rounded and strongly denticulate, largest denticles in posterior half. Basal pit well-marked. Pronotal puncturation relatively thick $(\varnothing=20-25 \mu \mathrm{m})$, but almost hidden behind a long and thick pubescence $(\mathrm{L}=45-80 \mu \mathrm{m})$ moderately raised. Transverse and flat scutellar shield. Elytra together oval and short, approximately 1.3 times longer than broad. Lateral margin slightly marked in anterior end. Strong strial puncturation $(\varnothing=34-40$ $\mu \mathrm{m})$. Elytral pubescence very long $(\mathrm{L}=90-130$
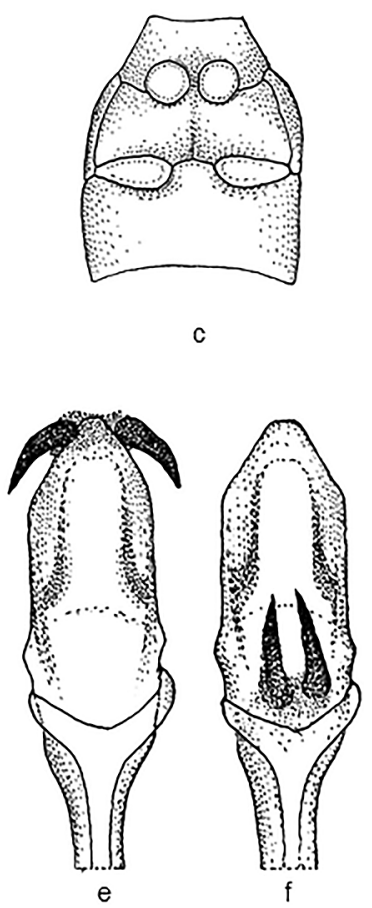

Fig. 18. Corticaria sylvicola C. N. F. Brisout de Barneville. - a. Dorsal habitus. - b. Antenna. - c. Ventrite 1. - d. Protibiae, male. - e. Internal sac. $-f$. Aedeagus, dorsal view.

$\mu \mathrm{m})$, thick, relatively raised and overlapping. Prosternum with two precoxal pits. Anterior tibiae depicted in Fig. 18d.

Abdomen. Ventrite 1 (Fig. 18c) almost twice as long as metasternite; 2 to 4 shorter and subequal; 5 longer than previous ones, rounded in posterior margin.

Aedeagus (Fig. 18e, f). Long ( $\mathrm{L}=0.700 \mathrm{~mm}$ ), broad and progressively sharp towards anterior third which extends to a prominent apex with rounded end. Internal sac with only two large curved rods $(\mathrm{L}=75-80 \mu \mathrm{m})$.

Distribution. S France, N Spain (Bouget \& Vincent 2008, Johnson 2007, Rücker 2013a).

Biology. This species has been found on piles of dry wood.

\subsection{Corticaria tarragonensis Dajoz (Fig 19)}

Corticaria tarragonensis Dajoz 1970: 260

Corticaria besucheti Dajoz 1970: 260

Type material examined. Holotype (đ): Spain. Tarragona, Sierra de Montsaut, 23.III.1959 (Leg. C. Besuchet).

Other specimens examined. Spain. Alicante, El Campello, 27.III.1978, 1 ex (leg. L. Subias). 
Fig. 19. Corticaria tarragonensis Dajoz.

-a. Dorsal habitus. - b.

Antenna. - c. Aedea-

gus, dorsal view. - d.

Aedeagus, lateral view.
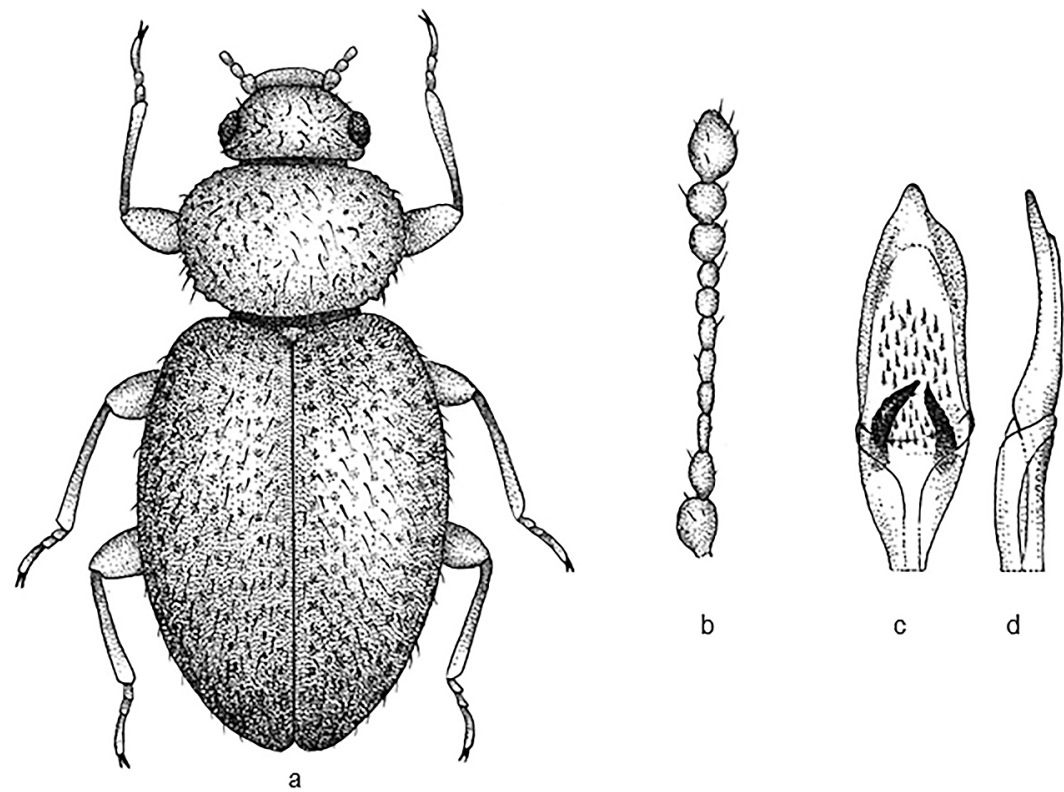

b

d
Madrid, Robledo de Chavela, 14.X.1973, 2 exx (leg. S. Pérez). Murcia, El Moral, 8.VI.1979, 1 ex (leg. J. J. Presa). Segovia, El Espinar, 21.XII.1977, 1 ex (leg. J. Berzosa). Teruel, Albarracín, 22.V.1982, 1 ex (leg. R. Outerelo) (USC).

Morphology. Body. Length 1.6-1.8 mm, convex (Fig. 19a). Testaceous yellow. Long yellowish pubescence. Metathoracic wings absent.

Head. Transverse (WL $=1.9-2.0$ ), softly punctured and reticulate. Eyes protuberant $(\mathrm{E}=$ 0.9-1.0), made up of a small number of thick ommatidia. Angular temples two thirds as long as eye length with forward-directed setae. Antennae long $(\mathrm{L}=0.621 \mathrm{~mm})$ (Fig. 19b), almost reaching posterior margin of pronotum. Antennomere I thick and dilated, II narrower than I, but almost twice as wide as III; III to VII longer than broad and progressively shorter than previous one; VIII as long as broad; IX subconical; X slightly transverse; XI longer than previous ones and obliquely truncated in apex.

Pronotum (Fig. 19a). Oval, moderately transverse $(\mathrm{WL}=1.5-1.6)$. Basal pit well-marked or barely marked. Strong and spaced puncturation. Largest width from anterior to middle part of pronotum, lateral margin with strong denticulation in posterior half, ending in two large teeth that mark the angles. Transverse and flat scutellar shield. Elytra together very convex and short (1.4 times longer than broad). Lateral margin visible in anterior half. Striae strongly punctured; thick and almost adjacent punctures. Anterior tibiae straight and obliquely truncated at base on external margin. First tarsomere dilated and ciliated in its inferior margin. Abdomen. Ventrite 1 twice as long as prosternum, with weak and spaced puncturation; ventrite 5 with a small pit.

Aedeagus (Fig. 19c, d). Sharp, with rounded apex. Internal sac with two large fang-shaped rods and a large number of smaller and needleshaped ones.

Distribution. E Spain (Dajoz 1970, Johnson 2007, Rücker 2013a).

Biology. Unknown.

\subsection{Corticaria tuberculata Dajoz (Fig. 20)}

\section{Corticaria tuberculata Dajoz, 1970: 264}

Type material examined. Holotype $(\circlearrowleft)$ : Spain. Cantabria, Espinama (leg. H. Franz) (NMW).

Morphology. Body. Length $1.9 \mathrm{~mm}$, oval and convex (Fig. 20a). Dark testaceous brown. Yellowish pubescence short and raised. Metathoracic wings absent.

Head. With spaced and shallow puncturation; dorsal surface slightly reticulate. Small eyes made up of large facets. Temples well-developed, as long as half of eye. Short antennae (Fig. 20b), 


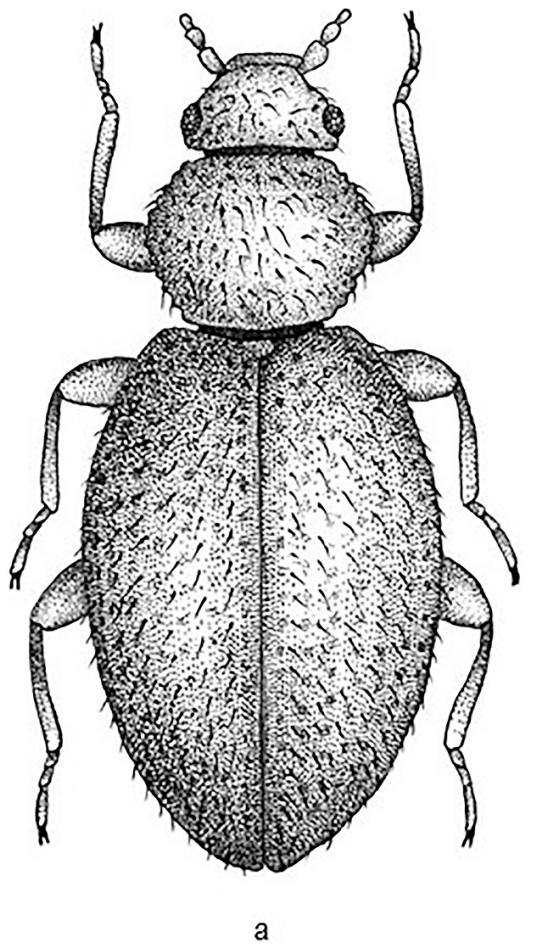

almost reaching base of pronotum. Antennomere I dilated; II narrower than I, but broader than III; III to VII clearly longer than broad, however, progressively shorter; VIII almost as wide as long; IX longer than broad; $\mathrm{X}$ narrower and shorter than IX, XI long and rounded in apex.

Pronotum (Fig. 20a). Transverse. Thick puncturation, arranged in a compact but rather shallow way. Basal pit barely marked; lateral margins denticulate, in shape of a regular curve. Lateral denticles better marked than in posterior part. Small scutellar shield, barely transverse or carenate. Elytra together very convex, oval, rounded in apical area and 1.4 times longer than broad. Humeral callus well-marked; dorsal surface covered in a short and raised pubescence both on striae and interstriae, which are flat. Striae with strong and compact puncturation, regular along their whole length. Lateral margin of elytra only visible in most anterior part. Prosternum lacking pits. Metasternum two thirds as long as that of first abdominal ventrite. Anterior tibiae with a tooth in internal apical angle. Internal margin straight and ciliated in its distal half.

Abdomen. Ventrite 5 (Fig. 20c) with a small tubercle protruding from medial area.

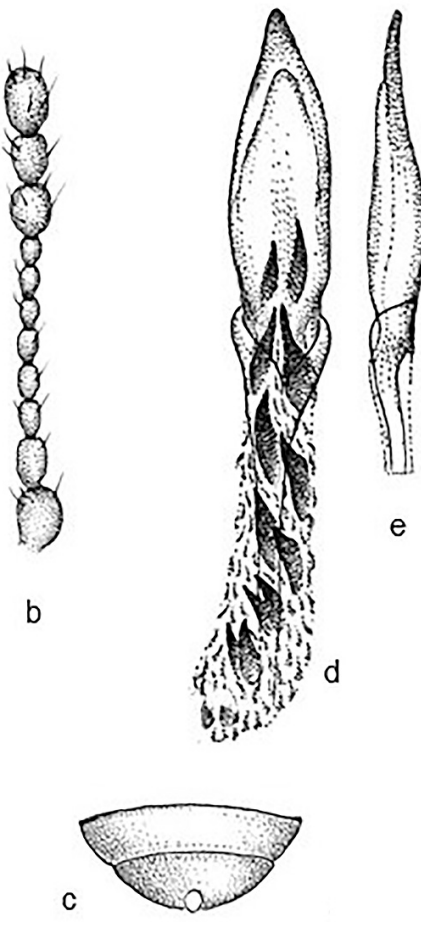

Fig. 20. Corticaria tuberculata Dajoz.

-a. Dorsal habitus.

- b. Antenna. - c.

Ventrite, male. $-d$. Aedeagus, dorsal view. - e. Aedeagus, lateral view.

Aedeagus (Figs 20d, e). Slightly curved in lateral view and regularly narrowed towards apex in dorsal view. Internal sac strongly developed with a dozen fang-shaped thick rods surrounded by a large number of smaller needle-shaped ones.

Distribution. NW Spain (Johnson 2007, Rücker 2013a).

Biology. Unknown.

\subsection{Corticaria tunisiensis $\mathrm{H}$. Brisout de Barneville (Fig. 21)}

Corticaria tunisiensis H. Brisout de Barneville 1884: 113

Type material examined. Lectotype ( $\lesssim$ ) (by present designation). Paralectotypes ( $2 \hat{\delta} \bar{O}$ and 3 우오) (by present designation). Mounted on same card: Syntype from Tunisia (leg. E. Reitter) (MNHN).

Morphology. Body. Length 1.8-1.9 mm, oval and convex (Fig. 21a). Yellowish grey-brown. Pubescence double and long $(\mathrm{L}=0.052-0.099$ $\mathrm{mm})$. Metathoracic wings absent.

Head. Transverse $(\mathrm{WL}=1.8)$, slightly narrower than pronotum. Shallow puncturation; 
Fig. 21. Corticaria tunisiensis $\mathrm{H}$. Brisout de Barneville. - a. Dorsal habitus. - b. Antenna. - c. Protibiae, male. $-\mathrm{d}$. Aedeagus, dorsal view. - e. Aedeagus, lateral view.

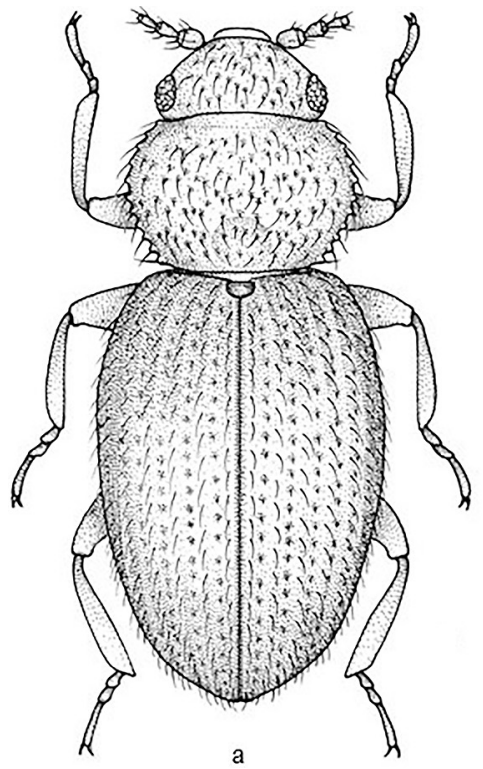

a
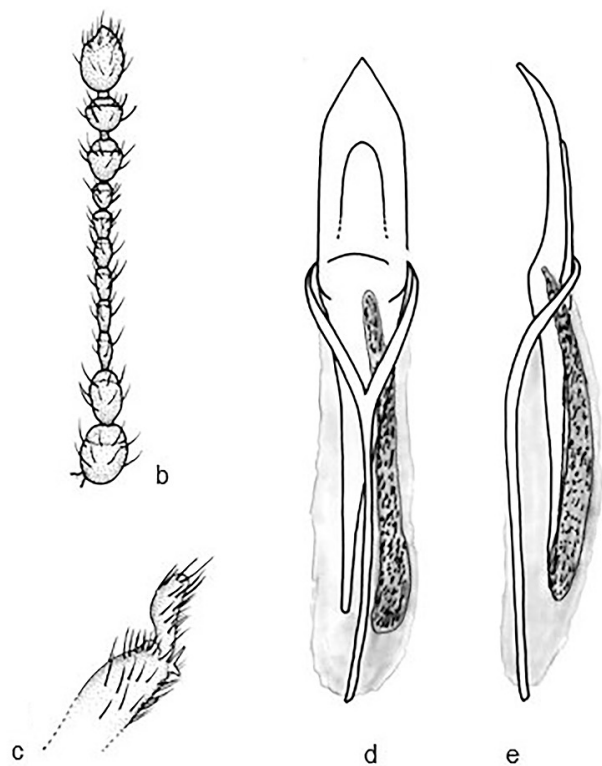

e

\section{Key to the species of the sylvicola group of Corticaria of Mediterranean region}

1. Antennomeres III to VIII longer than broad (Figs 1b, 12b, 18b)

- At least VIII antennomere not as long as broad (Figs 4b, 11b)

Long antennae $(\mathrm{L}=0.713 \mathrm{~mm})$, reaching base of pronotum (Fig. 21b). Antennomeres III to VII longer than broad; VIII as long as broad; antennomeres of club scarcely broader than long.

Pronotum (Fig. 21a). Moderately transverse $(\mathrm{WL}=1.6-1.7)$. Rounded lateral margins provided with 6-7 strong denticles, each with a long seta. Post-median depression well-marked. Strong and dense puncturation; punctures separated by a shorter distance than their diameter $(\varnothing$ $=16-18 \mu \mathrm{m})$. Transverse and carenate scutellar shield. Elytra together elongated, 3.1 times longer than pronotum. Puncturation more dispersed than that of pronotum; punctures separated by a larger distance than their diameter $(\varnothing=0.028$ $0.030 \mathrm{~mm}$ ). Male protibiae and mesotibiae with a forked spine in their internal apical end (Fig. 21c). Aedeagus (Fig. 21d, e). Elongated, broad with triangular apical end. Preputial sac with numerous small denticles.

Distribution. Tunisia (Johnson 2007, Rücker 2013a).

Biology. Unknown.

2. Elytral pubescence long and straight (Figs 12a, 18a)

- Elytral pubescence shorter and more recumbent (Figs 1a, 20a)

3. Male tibiae without spines in internal apical margin (Fig. 18d). Ventrite 5 of male with a deep semicircular pit. Aedeagus (Fig. 18e, f) broad and progressively sharp from anterior third to apex, prominent and with tip rounded. Internal sac with exactly sclerotized rods (Fig. 18f). $\mathrm{L}=1.5-1.7 \mathrm{~mm}$. Southern France, Spain

C. sylvicola Brisout de Barneville, 1863

- Male tibiae with a spine in internal apical margin. Aedeagus ( $\mathrm{L}=0.370 \mathrm{~mm}$ ). (Fig. 12d, e), with apex rounded and symmetrical; in lateral view, it forms an obtuse angle. Internal sac (Fig. 12d) with two large conical sclerotized rods $(\mathrm{L}=63 \mu \mathrm{m})$ and a large number of smaller ones with needle shape. $\mathrm{L}=1.6-1.8$ mm. Spain

C. lucasi Otero, López \& Rücker, 2013

4. Elytra widest in anterior third. Striae strongly punctured, almost continuous and badly 
aligned. Antennomeres VI and VII strongly narrowed at their base (Fig. 1a). Ventrite 5 of male (Fig. 1d) prolonged in a rounded and forward-curved tip. Aedeagus (Fig. 1e, f) asymmetric, lanceolate and sharp. Internal sac with two large sclerotized rods, surrounded by many other small and acicular ones. $\mathrm{L}=1.8-2.0 \mathrm{~mm}$. Caucasus, Spain and Turkey C.abdominalis Dajoz, 1970

- Elytra together broader in middle. Striae strongly punctured, with punctures dense and well aligned. Antennomeres VI and VII (Fig. 20b) not narrowed at their base. Ventrite 5 of male with a small median tubercle (Fig. 20c). Aedeagus (Fig. 20d, e). L=1.9 mm. Spain

C. tuberculata Dajoz, 1970

5. Elytra with lateral edge prominent 6

- Elytra with lateral edge faint or not pronounced, almost invisible in dorsal view except at frontal end

6. Convex body. Antennomeres VII and VIII (Fig. 4b) subquadrate. Pronotal lateral margin with 8-9 conspicuous denticles. Elytron lateral edge raised at its frontal end. Aedeagus (Fig. 4d) lanceolate, apex truncated. Internal sac (Fig. 4c) with a very large spine, accompanied by two lines of sclerotized smaller ones. $\mathrm{L}=1.8-2.0 \mathrm{~mm}$. Algeria, Spain

C. convexa Reitter, 1881

- Moderately flat body. Antennomeres VII and VIII subquadrate (Fig. 11b). Pronotal lateral margin with 8-9 conspicuous denticles (Fig. 11a). Elytra widest at their midlength. Lateral margin well marked anteriorly, with conspicuous shoulders. Aedeagus (Fig. 11d) elongated with a small and short apical petiole with a semicircular dilation at end. Length around $1.8 \mathrm{~mm}$. Spain

C. johnsoni Mariño, López \& Otero, 2007

7. Post-median depression of pronotum absent 8

- Post-median depression of pronotum wellmarked

8. Antennomeres III to VII clearly longer than broad

- Antennomeres III to V longer than broad, VI and VII broader than long

9. Pronotum very convex and transverse (WL $\geq$ 1.5)

- Pronotum (Fig. 5a) cordiform, little transverse $(\mathrm{WL} \leq 1.5)$
10. Aedeagus (Fig. 5c, d) sharp and truncated in apex. Internal sac with numerous very small sclerotized rods. $\mathrm{L}=1.8-2.0 \mathrm{~mm}$. Spain

C. cotovillae Otero \& Díaz Pazos, 1986

- Aedeagus (Fig. 13b) triangular with apex rounded. Sinuate in lateral view (Fig. 13c). Apex forms an obtuse angle in its apical section. Internal sac with numerous small sclerotized rods. Turkey

C. meybohmi Reike, 2015

11. Antennomere II three times longer than broad (Fig. 9e). Aedeagus (Fig. 9c, d) with apex retracted and sharp in lateral view. Internal sac (Fig. 9c) with two large sclerotized rods, and in middle of them a large number of smaller ones. $\mathrm{L}=1.6-1.7 \mathrm{~mm}$. Spain

$$
\text { C. franzi Dajoz, } 1969
$$

- Antennomere II two times longer than broad

12. Long temples ( $2 / 3$ of length of eyes). Long antennae, reaching base of pronotum. Ventrite 5 excavated. Aedeagus (Fig. 19c, d) sharp and with apex rounded. Internal sac with two fang-shaped sclerotized rods. $\mathrm{L}=1.6-1.8$ $\mathrm{mm}$. Morocco and Spain

C. tarragonensis Dajoz, 1970

- Temples short, almost absent. Antennae (Fig. 3b) short, not reaching base of pronotum. Ventrite 5 flat in both sexes. Aedeagus (Fig. $3 d$, e) triangular and with apex rounded. Internal sac (Fig. 3d, e) with two long sclerotized rods curved at end, towards centre of aedeagus. $\mathrm{L}=1.6-1.7 \mathrm{~mm}$. Spain

C. antonioi Otero, López \& Rücker, 2013

13. Body elongated (Fig. 14a). Pronotum clearly narrower than elytra. Antennomeres of club longer than wide (Fig. 14b). Aedeagus (Fig. $14 \mathrm{c}, \mathrm{d})$ with apex retracted; internal sac with three sclerotized rods (Fig. 14e). $\mathrm{L}=1.4-1.6$ mm. Southern France, Spain

C. pinicola Brisout de Barneville, 1866

- Body convex. Pronotum almost as broad as elytra. Antennomeres of club as long as broad, not transverse (Fig. 7b). Aedeagus (Fig. 7d, e) lanceolate with two lines of spines in internal sac. $\mathrm{L}=1.6-1.8 \mathrm{~mm}$. Algeria, Morocco, Spain, Tunisia C. diecki Reitter, 1875

14. Ventrite 5 elongated forming a spoon-shaped dilatation, in both sexes (Fig. 15c). Elytral punctures regularly aligned in striae. Aedea- 
gus (Fig. 15d, e) parallel with an apical narrowing; internal sac with three large sclerotized rods and numerous small ones. $\mathrm{L}=$ $1.6 \mathrm{~mm}$. Spain C.punctata Dajoz, 1970

- Ventrite 5 flat in both sexes, or with a deep pit in males

15. Antennomeres of club slightly broader than long. Pronotum strongly punctured. Ventrite 5 of males with a deep semicircular pit (Fig. 8c). Aedeagus (Fig. 8d). Internal sac with large curved sclerotized rods (Fig. 8e). L = 1.9-2.0 mm. Spain

$$
\text { C. espanyoli Otero \& López, } 2009
$$

- Ventrite 5 flat

16. Antennomeres of club clearly longer than broad (Fig. 16b). Margins of pronotum rounded, with 6-8 well-marked denticles. Elytral punctures disposed in irregular striae. Aedeagus (Fig. 16e) elongated with a small and very long apical petiole with a very little semicircular dilation at end. Two lines of sclerotized rods in internal sac (Fig. 16e). L= $1.7-1.8 \mathrm{~mm}$. Spain

C. rueckeri Otero, Mariño \& López, 2006

- Pronotum with at least 8-9 small teeth in margin. Short and oval elytra

17. Pronotum very transverse $(\mathrm{WL}=1.8)$ (Fig. 21a). Temples very short. Antennomere VIII as long as broad; IX and X slightly longer than broad. Elytral pubescence long $(\mathrm{L}=0.090$ $0.110 \mathrm{~mm}$ ) and recumbent. Aedeagus (Fig. 21d, e) elongated, broad with triangular apical end. Internal sac with many smaller denticles. $\mathrm{L}=1.8-1.9 \mathrm{~mm}$. Tunisia

C. tunisiensis H. Brisout de Barneville, 1884

- Pronotum longer than broad

18. Temples well marked, somewhat short, about a third length of eyes

- Temples well developed, about half length of eyes

19. Antenomere VIII broader than long, IX distinctly, X more obviously, broader than long. Pronotum with 6-7 coarse crenulations; front and hind angles strongly toothed due to size and shape of crenulations. Aedeagus (Fig. 2c, d) $(\mathrm{L}=0.500 \mathrm{~mm})$ strangulated in anterior third with elongated apical end. $\mathrm{L}=1.4-1.7$ $\mathrm{mm}$. South of Turkey, introduced in England

C. anatolica Johnson, 1989

- Antennomere VIII broader than long, IX clearly, X more obviously, broader than long (Fig. 6b). Pronotum with seven rather coarse crenulations between which a few minor ones. Aedeagus (Fig. 6c, d) with triangular apex and rounded margins. $\mathrm{L}=1.4-1.5 \mathrm{~mm}$. Crete

C. cretica Johnson, 1989

20. Pronotum with at least eight rather weak crenulations (Fig. 17a). Aedeagus (Fig. 17c, d) with triangular apex. $\mathrm{L}=1.6-1.8 \mathrm{~mm}$. Morocco C. suspecta Johnson, 1989

- Pronotum with 8-9 moderate sized crenulations (Fig. 10a). Aedeagus (Fig. 10c, d) with pointed end. $\mathrm{L}=1.6-1.8 \mathrm{~mm}$. Morocco C. fulvoides Johnson, 1989

Acknowledgements. The authors are deeply indebted to: Nicole Berti, (died 2008) Muséum National d'Histoire Naturelle, Paris, France, who loaned types from the Belon/Pic collection; Dmitri Logunov, The Manchester Museum, Manchester, United Kingdom; Harald Schillhammer, Naturhistorisches Museum Wien, Austria; who kindly loaned type material; Azadeh Taghvian, Muséum National d'Histoire Naturelle, Paris, France, who loaned types from the Belon/Pic collection and Brisout collection.

\section{References}

Aubé, C. 1866: Description de nouvelles espèces de coléoptères de France. - Annales de la Société Entomologique de France (4) 6: 161-164

Belon, M. J. 1897: Essai de classification générale des Lathridiidae avec le catalogue systématique et alphabétique de toutes les espèces du globe. - Revue d'Entomologie 16: 105-221.

Bouget, C. \& Vincent, R. 2008: Les Latridiidae de la faune de France continentale et de Corse: mise à jour de la clé de genres et du catalogue des espèces (Coleoptera, $\mathrm{Cu}$ cujoidea). - Bulletin de la Société entomologique de France 113: 101-120.

Brisout de Barneville, C. N. F. 1863: [new taxa]. — In: Grenier, A. (ed.), Catalogue des coléoptères de France et matériaux pour servir à la faune des coléoptères françaises. Paris, L. Toinon IV: 3-79.

Brisout de Barneville, C. N. F. 1866: Coléoptères nouveaux trouvés en Espagne pendant l'excursion de la Société en 1865. - Annales de la Sociétè Entomologique de France (4) 6: 355-426.

Brisout de Barneville, H. 1884: [new taxa]. — Bulletin de la Société Entomologique de France 12: 113-114.

Dajoz, R. 1969: Étude des Corticaria du groupe sylvicola $\mathrm{Ch}$. Brisout et description d'une espèce nouvelle (Coléoptères, Lathridiidae). - Revue d'Écologie et Biologie du Sol VI-(1): 93-97.

Dajoz, R. 1970: Contribution à l'étude des Coléoptères Lathridiidae de la Péninsule Ibérique et du Maroc. - 
Revue d'Écologie et Biologie du Sol VII-(2): 255275.

Johnson, C. 1989: Studies of the genus Corticaria Marsham (Coleoptera, Latridiidae), part. 3. - Entomologist's Gazette 40: 79-90.

Johnson, C. 2007: Latridiidae, 635-649. — In: Löbl, I. \& Smetana, A. (eds), Catalogue of Palaearctic Coleoptera 4. Apollo Books. Stenstrup, Denmark, 935 pp.

Mariño, P., López M. J. \& Otero J. C. 2007: Corticaria johnsonii, a new species of the sylvicola group within the genus Corticaria Marsham, 1802 (Coleoptera: Latridiidae). — Entomologica Fennica 18: 24-26.

Otero, J. C. \& Díaz Pazos, J. 1986: Lathridiidae de la Península Ibérica. IV: Descripción de Corticaria cotovillae n. sp. (Coleoptera). - Nouvelle Revue d'Entomologie 3(2): 167.

Otero, J. C. 1997: Three new species and distributional records of Micrambe C. G. Thomson, 1863 and Cryptophagus Herbst, 1792 (Coleoptera: Cryptophagidae) from Israel and Turkey. - Revue Suisse de Zoologie 104(1): 207-216.

Otero, J. C. 2001: Records of Cryptophagus Herbst, 1792 from the Himalaya, with a new species from Thailand (Coleoptera: Cryptophagidae). - Revue Suisse de Zoologie 108(4): 987-992.

Otero, J. C., Mariño, P. \& López, M. J. 2006: Anew Iberian species of Corticaria Marsham in the C. sylvicola group (Coleoptera: Latridiidae). — Koleopterologishe Rundschau 76: 373-377.

Otero, J. C. \& López M. J. 2009: Corticaria espanyoli sp. n. (Coleoptera, Latridiidae, a new Iberian species of the sylvicola group. - Deutsche Entomologische Zeitschrift 56(2): 267-269. DOI 10.1002/mmnd. 200900023.

Otero, J. C., López M. J. \& Rücker W. H. 2013: Review of the Corticaria sylvicola group (Coleoptera: Latridii- dae), with a description of two new species from the Iberian Peninsula. - Annales de la Société entomologique de France 49(3): 233-239. Available from http://dx.doi.org/10.1080/00379271.2013.854090 (accessed 13 September, 2016).

Otero, J. C. \& López, M. J. 2016: Coleoptera Latridiidae. — In: Ramos, M. A., Alba-Tecedor, J., Bellés, X., Gosálbez, J., Guerra, A., Macpherson, E., Serrano, J \& Templado, J. (eds), Fauna Ibérica 42: 1-288. Museo Nacional de Ciencias Naturales. CSIC. Madrid, Spain.

Reike, H. 2015: Eine neue Art der Gattung Corticaria aus der sylvicola-Gruppe und eine neue Corticarina (Coleoptera, Latridiidae). - Mitteilungsblatt für Systematik und Taxonomie der Latridiidae (Insecta: Coleoptera) $18: 1-4$.

Reitter, E. 1875a: Revision der europäischen Lathridiidae. - Stettiner Entomologische Zeitung 36: 297-340.

Reitter, E. 1875b: Revision der europäischen Lathridiidae. - Stettiner Entomologische Zeitung 36: 410-445.

Reitter, E. 1881: Bestimmungs-Tabellen der europäischen Coleopteren. III Heft. I Auflage. Enthaltend die Familien: Scaphidiidae, Lathridiidae und Dermestidae. Verhandlungen der Königlich-Kaiserlichen Zoologisch-Botanischen Gesellschaft in Wien 30: 41-94.

Rücker, W. H. 2013a: Coleoptera, Latridiidae. - In: Audisio, P. (ed.), Fauna Europaea: Coleoptera, Beetles, Fauna Europaea version 2.6.1, Available from http://www.faunaeur.org (accessed 13 September, 2016).

Rücker, W. H. 2013b: Studien von Typenmaterial der Gattung Corticaria Marsham, 1802 mit Festlegung von Lectotypen aus den Sammlungen C. Brisout de Barneville und H. Franz (Coleoptera, Latridiidae). - Mitteilungsblatt für Systematik und Taxonomie der Latridiidae (Insecta: Coleoptera) 10: 27-30. 